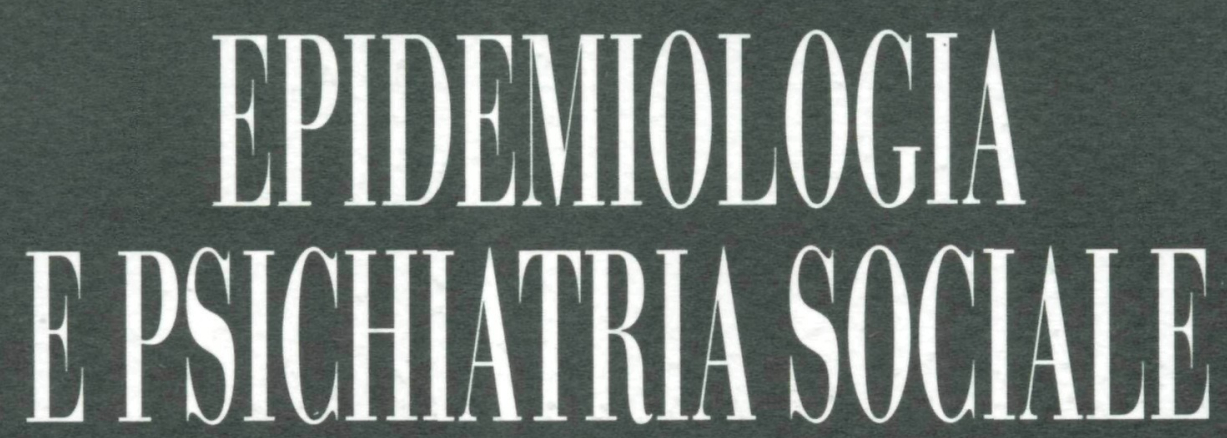

Volume 6, Numero 2, Maggio-Agosto 1997

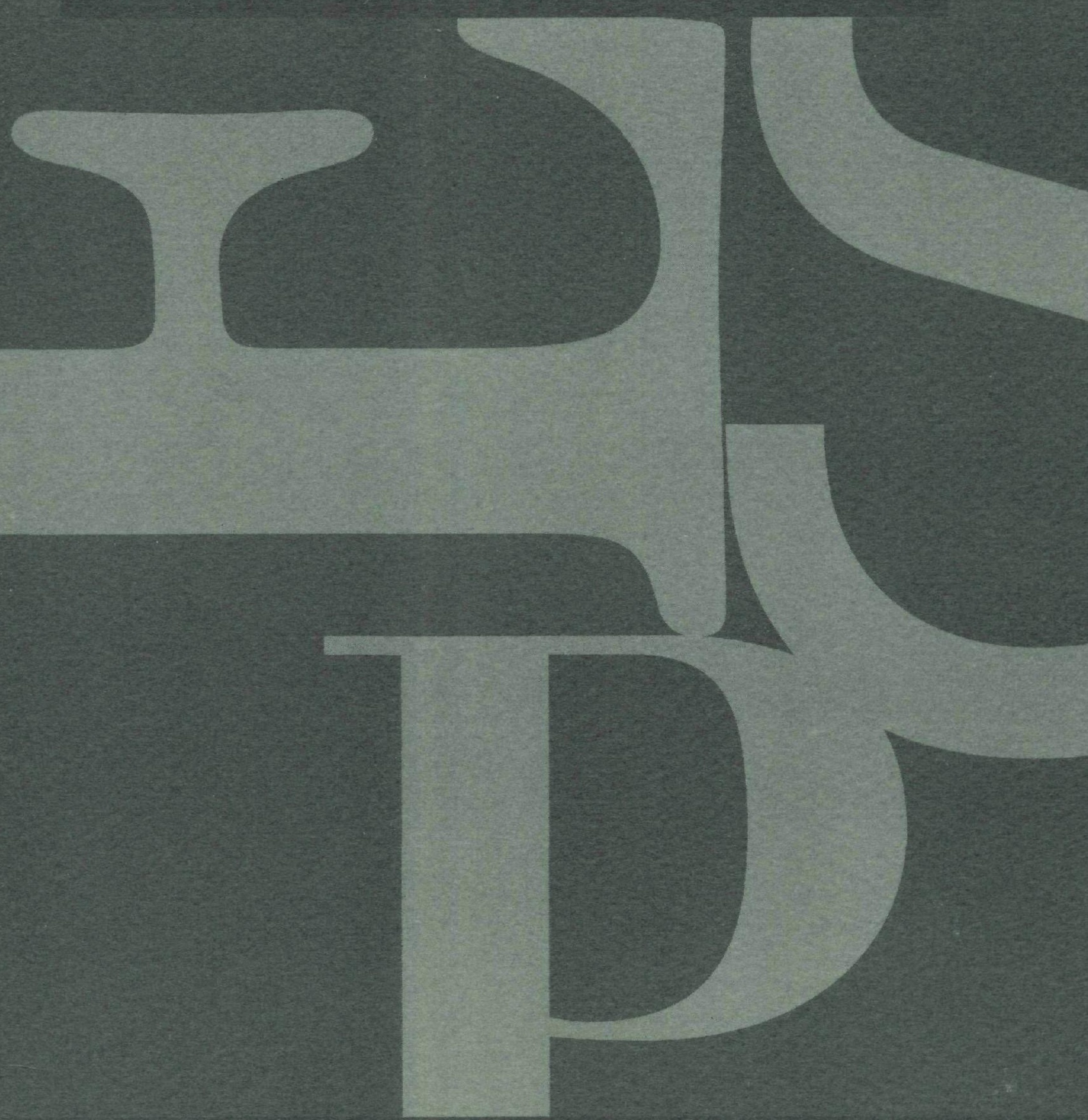




\section{SCELTA TERAPEUTICA}

\section{AD AMPIO SPETTRO DI IMPIEGO}
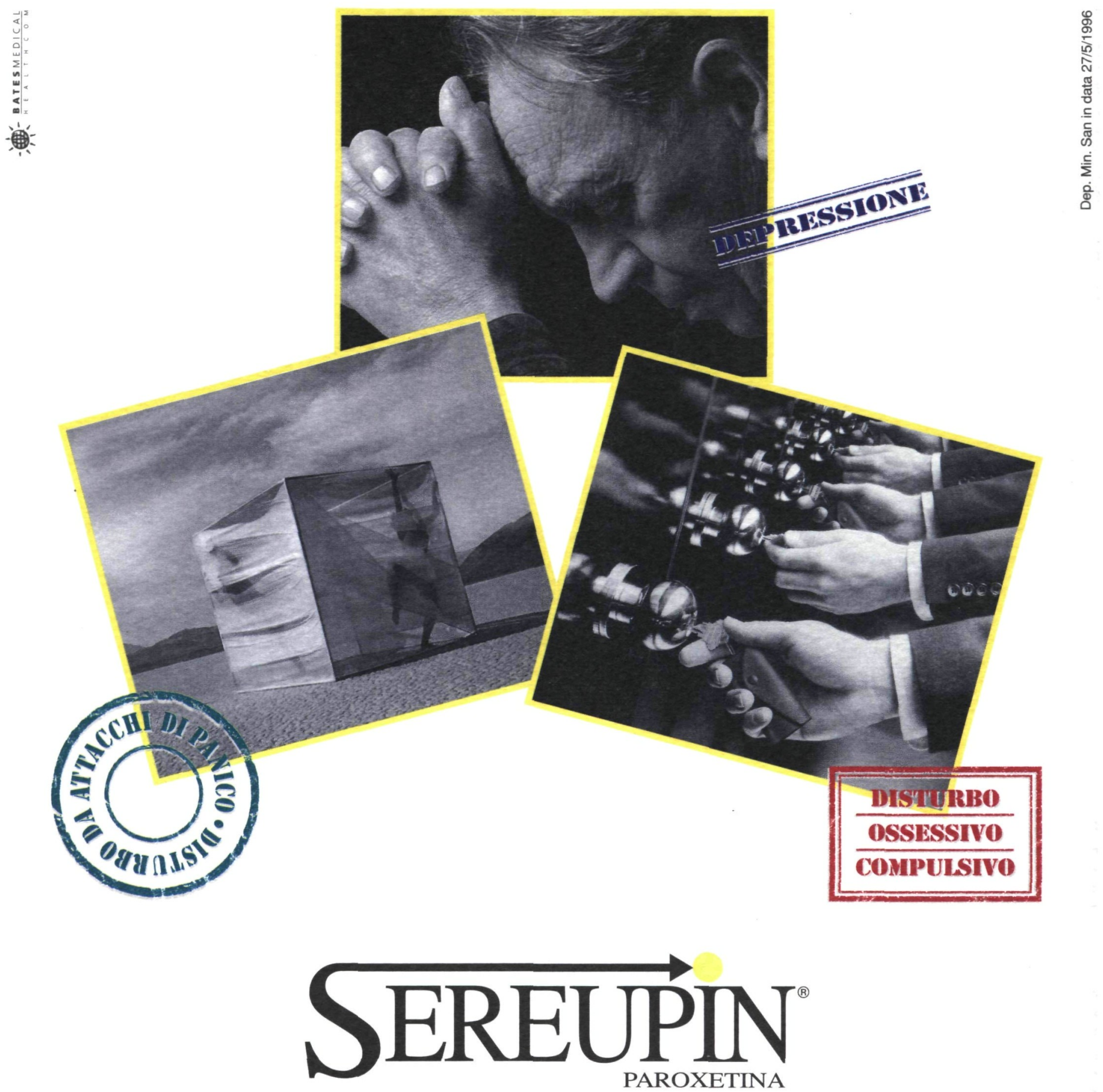

EFFICACIA ANTIDEPRESSIVA ESTESA ALLE DIVERSE SITUAZIONI CLINICHE 


\section{EPIDEMIOLOCIA EPSICHATTRIA SOCIALE}

Direttore

Michele Tansella

\section{Comitato Direttivo}
A. Balestrieri (Verona)
M. Balestrieri (Catania)
C. Bellantuono (Verona)
G. Berti-Ceroni (Bologna)
A. Biggeri (Firenze)
G. de Girolamo (Bologna)
C. Faravelli (Firenze)

G. Ferrari (Bologna)

T. Losavio (Roma)

M. Maj (Napoli)

A. Marinoni (Pavia)

P. Martini (Arezzo)

R. Micciolo (Trento)

G. Muscettola (Trieste)

P. Morosini (Roma)

P. Pasquini (Roma)

M. Ruggeri (Verona)

B. Saraceno (Milano)

E. Torre (Pavia)

F. Veltro (Benevento)

Ch. Zimmermann (Verona)

\section{Comitato Internazionale di Consulenza}

B. Cooper (London)

B. P. Dohrenwend (New York)

R. Giel (Groningen)

Sir D. Goldberg (London)

A. S. Henderson (Canberra)

A. Jablensky (Perth)

J. Leff (London)

A. Lesage (Montreal)

P. Munk-Jørgensen (Aarhus)
E S. Paykel (Cambridge)

D.A. Regier (Rockville)

L. N. Robins (St. Louis)

N. Sartorius (Geneva)

G. Thornicroft (London)

J. L. Vazquez-Barquero (Santander)

M. M. Weissman (New York)

G. Wilkinson (Liverpool)

H. U. Wittchen (Munich)

\section{Redattore-Capo e Responsabile dei rapporti con l'Editore}

R. Fianco (Verona)

\section{Gruppo di produzione editoriale}

Direttore responsabile: Francesco De Fiore; Produzione: Roberto Bonini

Pubblicità: Luciano De Fiore, Barbara Vella, Lorenza Bonaccorsi; Abbonamenti: Roberto Ciapponi Fotocomposizione e stampa: Grafica 2000, Città di Castello (Perugia)

\section{Questa rivista è recensita in: \\ Excerpta Medica/EMBASE \\ Faxon Finder \\ Faxon XPRESS \\ Index Medicus/MEDLINE \\ Lettera (Ist. Mario Negri) \\ Mental Health Abstracts \\ Sociological Abstracts}

Sommario e informazioni su EPS

su Internet, alla pagina

http://www. medicina.univr.it/ psymed/eps.htm

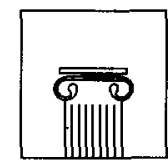

\section{Il Pensiero Scientifico Editore}




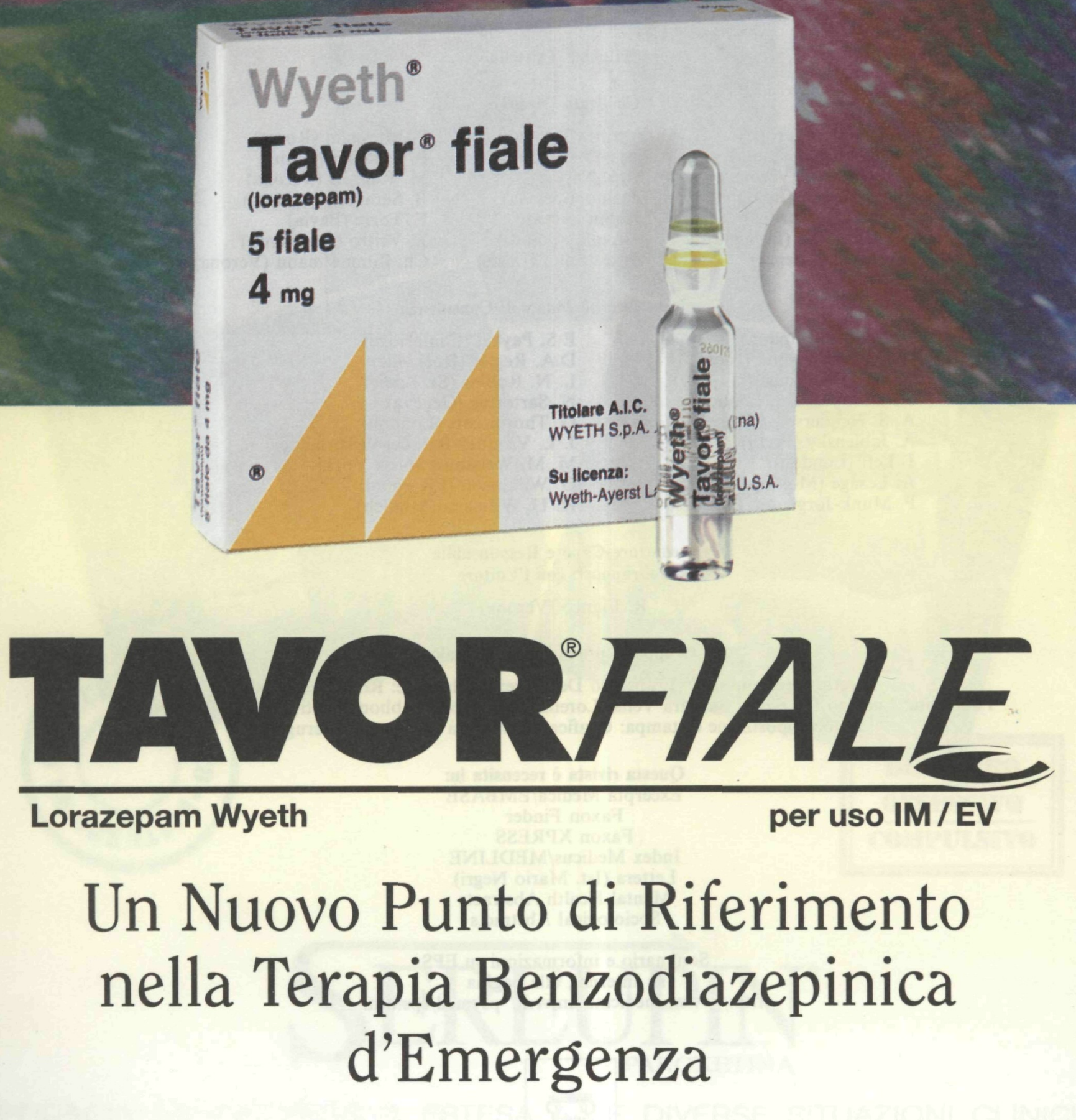

Wyeth 


\section{EPIDEMIOLOCJA \\ EPSSCHIATRIA SOCIALE}

Volume 6, Numero 2, Maggio-Agosto 1997

\section{SOMMARIO}

Linee guida per la preparazione di curriculum vitae ed elenco pubblicazioni. Uniformare lo stile di presentazione per favorire una più omogenea valutazione dei titoli nei concorsi di psichiatria, M. Tansella, P. Pancheri, G.B. Cassano

\section{Editoriali}

La ricerca in psicoterapia: perché in Italia è così poca? G.A. Fava $\$ 81$

The efficacy of psychotherapy, J.C. Markowitz

I porcospini rivisitati, $R$. Carli

Psychotherapy research: linking efficacy to policy and practice, G. Parry, A.D. Roth

\section{Articoli}

M. Chiappelli, F. Pileggi, G. Rosini, G. Neri, I «pazienti quotidiani»: follow-up a tre anni di un gruppo di utenti alti utilizzatori di un centro di salute mentale cittadino

E. Guerriero, Servizi psichiatrici, cooperazione sociale, volontariato.

Nuovi rapporti per nuove strategie

E. Favaretto, S. Torresani, Il legame genitoriale come fattore predittivo dello sviluppo di disturbi psichiatrici in età adulta

G. Fattore, M. Percudani, C. Pugnoli, A. Contini, I costi delle prestazioni in psichiatria: un'analisi condotta in un Centro Psico-Sociale (CPS) pubblico in Lombardia 


\section{TAVORFIALE}

\section{RIASSUNTO DELLE CARATTERISTICHE DEL PRODOTTO}

I. DenOMINazIONe DELla SPECIALITA MEdicinale: taYor fiale 2. COMPOSIZIONE QUALI-QUANTITATIVA IN PRINCIPI ATTIVI ED ECCIPIENTI Ogni fiala contiene: lorazepam mg 4,00. Eccipienti: glicole polietilenico $400 \mathrm{mg}$ 202,50; alcool benzilico $\mathrm{mg}$ 20,80; glicole propilenico q.b. a $\mathrm{ml}, 1,0$. 3. FORMA FARMACEUTICA fiale per uso iniettabile i.m. o e.v. 4. INFORMAZIONI CLINICHE 4.I Indicazioni terapeutiche I) Nella premedicazione anestetica per alleviare l'ansia e per diminuire la rievocazione di eventi associati ad interventi chirurgic e diagnostici. Mon ci sono dati sufficienti a supporto dell'uso di Tavor fiale durante il travaglio e il parto, induso il taglio cesareo; pertanto l'uso del Tavor Fiale in queste situazioni non è raccomandato (vedere al paragrafo "Gravidanza e Allattamento").2) Per il sollievo sintomatologico dell'ansia acuta nevrotica o psicotica (è preferibile la somministrazione per via endovenosa).3) Per il trattamento degli stati epilettici dovuti a diversi tipi di epilessia parriale e generalizzata. I tipi di epilessia che hanno dimostrato nispondere a Tavor Fiale sono: epilessia generalizata (tonico-donica, "grande male"), epilessia da assenza generalizzata ("piccoto male") o stupor a punta-onda, epilessia pariale elementare ("motoria locale"), epilessia parziale complessa ("psicomotoria"), e associazioni quali epilessie generalizzate con inizio focale. II trattamento iniziale con Tavor Fiale porta alla cessazione prolungata dell'attività epilettica (ved. al paragralo "Awertenze"). 4.2 Posologia e modo di somministrazione DosugGlo I) Premedicazione anestetica Per un massimo effetto benefico, il dosaggio dovrebbe essere stabilito in base al peso corporeo (il dosaggio usuale va dai 2 ai $4 \mathrm{mg}$ ) e somministrato come segue:a) per via endovenosa: $0.044 \mathrm{mg} / \mathrm{kg}$ fino ad un totale di $2 \mathrm{mg}$, somministrati $15-20$ minuti prima dell'intervento chirurgico previsto per ottenere un effetto ottimale, misurato come vuoto di memoria.Questa dose per via endovenosa è sufficiente per sedare la maggior parte dei pazienti adulti, e normalmente non deve essere superata in pazienti con oltre 50 anni $d$ etz. Mei pazienti nei quali è preleribile ottenere un maggior grado di vuoto di memoria per eventi riguardanti Pintervento chirurgico, può essere somministrato un dosaggio maggiore, cioé $0.05 \mathrm{mg} / \mathrm{kg}$ fino ad un totale di 4 mg.Prima della somministrazione endovenosa di Javor Fiale deve essere immediatamente disponibile l'apparecchiatura necessaria a mantenere aperte le vie respiratorie.b) per via intramuscolare: 0.05 $\mathrm{mg} / \mathrm{kg}$ fino ad un massimo di $4 \mathrm{mg}$, somministrati almeno 2 ore prima dell'intervento chirungico previsto per ottenere un effetto ottimale, misurato come vuoto di memoria.Come con tutti i farmaci utilizzati nella premedicazione, la dose deve essere adattata in modo individuale. Nei pazienti anziani o debilitati, 0 in pazienti con funzione epatica o renale compromesse $o$ in quelli con serie patologie respiratorie o cardiovascolari, si raccomanda una riduzione del dosaggio. Hel caso di anestesia locale e di procedure diagnostiche richiedenti la collaborazione del paziente, può essere appropriato l'uso concomitante di un analgesico.l dosaggi di altri agenti depressivi del sistema nervoso centrale somministrati concomitantemente a Tavor Fiale devono di norma essere ridorti.Si raccomanda che ogni farmaco concomitante sia somministrato in siringhe separate. 2) Psichiatria Mei casi di ansia acuta con o senza agitazione psicomotoria (e a seconda della sua eziologia, gravità, e peso del paziente) la dose raccomandata iniziale $\dot{e}$ di $2-4 \mathrm{mg}$ e.v. 0 i.m., cooe $0.05 \mathrm{mg} / \mathrm{kg}$ (la somministrazione endovenosa è preferibile). Ove indicato, la dose può essere ripetuta dopo 2 ore. Una volta che la sintomatologia acuta è stata controllata, è necessario che il paziente riceva un appropriato trattamento per la condizione di base; Javor per uso orale puó essere preso in considerazione se è richiesto un ulteriore trattamento benzodiazepinico. 3) Stato epilettico dovuto a vari tipi di epilessia pariale e generalizata La dose iniziale usualmente raccomandata di Tavor Fiale ì di $4 \mathrm{mg}$ somministrati per iniezione endovenosa lenta (2 $\mathrm{mg}$ al minuto) per pazienti di 18 anni ed oltre. Se le convulsioni continuano o si verificano nuovamente dopo un periodo di osservazione di 10-15 minuti, può essere somministrata un'ulteriore dose di $4 \mathrm{mg}$ per via endovenosa. Se la seconda dose non portasse ad un controllo degli attacchi epiletrici dopo un altro periodo di osservazione di 10-15 minuti, devono essere impiegate altre misure per controllare 10 stato epilettico. Hel periodo di 12 ore può essere somministrato un massimo di $8 \mathrm{mg}$ di Tavor fiale. SomHNustuntone Non somministrare per via intra-arteriosa. Quando somministrato per via intramuscolare, Tavor Fiale, può essere iniettato non diluito in prolonditzi in un'appropriata massa muscolare. Comunque, poiché Tavor Fiale è un pó vischioso, liniezione intramuscolare puo essere facilitata diluendo it prodotto con un uguale volume di soluzione compatibile.Immediatamente prima dell'uso endovenoso, Tavor Fiale deve essere diluito con un uguale volume di soluzione compatibile. Quando opportunamente diluito, il tarmaco puó essere iniettato direttamente in vena o nel tubo di un'infusione endovenosa già esistente. La velocità di iniezione non deve eccedere i $2 \mathrm{mg} /$ minuto. Prima della somministrazione, le fiale di prodotti per uso parenterale devono essere con- trollate visivamente per quanto riguarda l'eventuale presenza di un precipitato o un'alteraxione del colore. Mon usare fiale in presenza di un'alterazione del colore o di un precipitato. Istruzioni per la diluizione nellluso endovenoso Aspirare la quantità desiderata di Tavor Fiale nella siringa, quindi aspirare lentamente il volume desiderato di diluente. Tirare indietro lentamente lo stantuffo per creare un ulteriore spazio per miscelare. Immediatamente miscelare accuratamente il contenuto capovolgendo delicatamente la siringa e ripetere fino ad ottenere una soluzione omogenea. Hon agitare vigorosamente in quanto questo può portare ad un inglobamento d'aria. Tavor fiale è compatibile con le seguenti soluzioni per diluvizione per almeno I ora:Acqua sterile per iniezione, U.S.P.Soluzione di donuro di sodio, U.S.P.Soluzione al $5 \%$ di destrosio, U.S.P.Soluzione batteriostatica per iniezione, U.S.P., con parabeniSoluzione batteriostatica per iniezione, U.S.P., con Alcool Benzilicosoluzione batteriostatica di doruro di sodio, U.S.P., con Alcool Benzilico 4.3 Controindicazioni l'uso di Tavor Fiale per via intra-arteriosa è controindicato perché, come con altre benzodiazepine iniettabili, liniezione intra-arteriosa può produrre uno spasmo dell'arteria che può portare a gangrena, per la quale può essere richiesta l'amputazione.Tavor fiale è controindicato nella sindrome apneica durante il sonno o nell'insufficienza respiratoria grave, nell' insufficienza epatica grave, nella miastenia grave e nel glaucoma ad angolo stretto, in gravidanza e nell'allattamento.la sicurezza e l'effi- cacia di Javor Fiale nei bambini non sono state stabilite e, pertanto, Puso del prodotto è generalmente controindicato.Tavor fiale è inoltre controindicato in pazienti con accertata ipersensibilità alle benzodiazepine, compreso il lorazepam o altre sostanze strettamente correlate dal punto di vista chimico, o agli eccipienti glicole polietilenico, glicole propilenico e alcool benzilico. 4.4 Speciali Avvertenze e Precauzioni per I'Uso Avvertenze Prima della somministrazione endovenosa Tavor Fiale deve essere diluito con una uguale quantità di solvente compatibile (ved. "Posologia e modo di somministrazione"). L'iniezione endovenosa deve essere praticata lentamente e con aspirazioni ripetute. E' necessario che l'iniezione non venga effettuata per via intra-arteriosa e che non si verifichi travaso perivascolare. L'ostruzione pariale delle vie aeree può verificarsi in pazienti fortemente sedati. Tavor Fiale, quando somministrato da solo per via endovenosa in quantità maggiore della dose raccomandata, oppure alla dose raccomandata insieme ad altri farmaci usati durante l'induzione dell'anestesia, può produrre forte sedazione. Pertanto, l'attrezzatura necessaria per mantenere le vie respiratorie aperte e supportare la respirazione/ventilazione deve essere disponibile e usata in caso di necessitàl pazienti devono essere avertiti che, poiché la propria tolleranza all'alcool o ad altri tarmaci depressori del SHC diminuisce con l'assunzione di Tavor Fiale, è necessario o evitare del tutto queste sostanze o diminuirne le dosi. Bevande alcooliche non devono essere consumate per almeno 24-48 ore dopo la somministrazione di Tavor fiale a causa degli effetti di potenziamento sulla depressione del SKC osservati con le benzodiazepine in generale.Si raccomanda di tenere sotto osservazione i pazienti che hanno ricevuto lavor fiale per 24 ore dopo l'ultima somministrazione. Quando Tavor Fiale viene usato per interventi brevi in pazienti ambulatoriali, i parienti devono essere accompagnati da una persona adulta responsabile quando lasciano Pambulatorio. I pazienti devono essere awertiti di non guidare 0 intraprendere attivita che richiedono una certa attenzione per $24-48$ ore dopo la somministrazione. Atterzzioni della performance possono persistere per intervalli più lunghi a causa dell'etá, dell'uso concomitante di altri farmaci, dello stress chirurgico o delle condizioni generali del paziente. Inoltre $i$ pazienti devono essere awertiti che la deambulazione prematura (entro $B$ ore dalla somministrazione del prodotto) può provocare cadute e conseguenti lesioni.Non ci sono dati sufficienti a supporto dell'uso di Tavor Fiale nelle procedure endoscopiche in pazienti ambulatoriali. Quando queste procedure vengono condotte in parienti ospedalizati, è necessario tenere i pazienti sotto stretto controllo e, prima dell'intervento, ridurre al minimo l'attività faringea riflessa somministrando un'adeguata anestesia da contatto o regionale.Hon ci sono prove a supporto dell'uso di Tavor Fiale nel coma o nello shockl'associazione di scopolamina e Tavor Fiale non è raccomandata, in quanto può causare un'aumentata incidenza di sedazione, allucinazioni e comportamento irrazionale (vedere "Interazioni"). Tavor Fiale non è raccomandato per il trattamento di mantenimento dell'epilessia. Dopo aver ottenuto il controllo degli attacchi associati allo stato epitettico, devono essere somministrati larmaci appropriati per la prevenzione di ulteriori attacchi. Mel trattamento dello stato epilettico dovuto ad uno squilibrio metabolico acuto reversibile (per es. ipoglicemia, ipocalcemia, iponatremia, ecc.) devono essere intraprese misure immediate per correggere il difetto specifico.Tavor fiale non è indicato per il trattamento primario di malattie psicotiche o disturibi depressivi e non deve essere usato da solo per il trattamento di pazienti depressi. l'uso delle benzodiazepine pùo avere un effetto disinibente e puo facilitare tendenze suicide in pazienti depressi.Non ci sono dati a supporto dell'uso prolungato di Tavor Fiale.La terapia con benzodiazepine deve essere interrotta gradualmen-

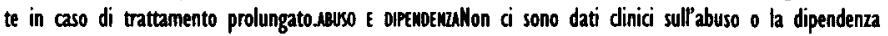
con Iavor Fiale. Comunque, sulla base delle esperienze con le benzodiazepine orali, $i$ medici devono tener presente che dosi ripetute di Tavor fiale somministrate per un periodo di tempo prolungato possono determinare dipendenza fisica e $/ 0$ psicologicall rischio di dipendenza aumentz con dosi elevate e con trattzmenti prolungati. E' inoltre maggiore nei parienti con precedenti di abuso di alcool o sostanze stupefacenti. Se si sviluppa dipendenza fisica, la sospensione brusca del trattamento puó essere associata a sintomi da sospensione. I sintomi riportati dopo sospensione di benzodiazepine orali indudono cefalea, dolore muscolare, ansia, tensione, depressione, insonnia, agitazione, confusione, irritabilità, sudorazione e la comparsa di fenomeni rebound, per cui $i$ sintomi che averano portato al trattamento con le benzodiazepine si ripresentano in forma accentuata. Può essere difficile distinguere questi sintomi da quelli originari per $i$ quali è stato prescritto il tarmaco. Nei casi gravi possono verificarsi i seguenti sintomi: derealizazione, depersonalizzazione, iperacusia, tinnito, intorpidimento e formicolio delle estremità, ipersensibiliti alla luce, ai rumori e ai contatti fisici, movimenti involontari, vomito, allucinazioni e comvulsioni. Le convulsioni possono essere più comuni in pazienti con pre-esistenti disturbi di natura epilettica oppure che tanno uso di altri tarmaci che abbassano la soglia convulsiva, quali gli antidepressivi.Sintomi da sospensione, specialmente i più gravi, sono più comuni in quei pazienti che hanno riceruto dosi elevate per un lungo periodo di tempo; essi possono però verificarsi anche dopo sospensione di benzodiazepine prese di continuo a dosaggi terapeutici, specie se la sospensione amiene in modo brusco. Poiché il rischio di sintomi da sospensione/fenomeni rebound è maggiore dopo internuzione brusca, le benzodiazepine devono essere interrotte gradualmente in caso di trattamento prolungato. Precauzioni Alcuni pazienti in trattamento con benzodiazepine hanno sviluppato discrasia ematica, e alcuni hanno aruto elevazioni degli enzimi epatici. Quando una terapia prolungata è dinicamente necessaria si raccomandano periodici controlli ematologici e della funzione epatical'ansia può essere il sintomo di diversi altri disturbi. Deve essere considerata la possibilità che l'ansia possa essere correlata ad un disturbo fisico o psichiatrico latente per il quale esiste un trattamento più specifico.Durante il trattamento con le benzodiazepine puó emergere una depressione pre-esistente (ved. "Awertenze") Amnesia transitoria anterograda o disturbi della memoria sono stati riportati concomitantemente all'uso di benzodiazepine. Questo effetto può essere vantaggioso quando il Tavor fiade viene utilizato nella premedicazione.Sebbene lipotensione si sia verificata solo raramente, te benzodiazepine devono essere somministrate con cautela in pazienti nei quali un abbassamento della pressione ematica può determinare complicazioni cardio- e cerebro-rascolari. Questo è particolarmente importante in pazienti anziani.Dilatazione esofagea si è verificata in ratti trattati con lorazepam per più di un anno con dosaggi di $6 \mathrm{mg} / \mathrm{Kg} / \mathrm{di}$. II dosaggio massimo privo di effetto è stato di $1.25 \mathrm{mg} / \mathrm{kg} / \mathrm{die}$, cioé approssimativamente 6 volte la dose terapeutica massimo nell'uomo che è di $10 \mathrm{mg}$ al giorno. L'effetto è stato reversibile soltanto se il trattamento è stato sospeso entro 2 mesi dalla prima osservazione del fenomeno. Hon 
si conosce il significato clinico di cio. Comunque, Puso di lorazepam per periodi prolungati e in pazienti geriatrici richiede cautela; inoltre sono necessạri frequenti controlli alla ricerca di sintomi di disturbi del tratto gastrointestinale superiore. l'uso di lorazepam per periodi prolungati non è raccomandato. Gruppi particolari di pazienti Come per altri farmaci utilizzati nella premedicazione anestetica, la massima attenzione deve essere usata nel somministrare tavor Fiale a pazienti anziani o molto malati e a quelli con limitata riserva polmonare o con labilità cardiocircolatoria, a causa della possibilita che possa verificarsi apnea e/o arresto cardiaco ipossico. Un'attrezzatura di rianimazione per la ventilazione assistita deve essere immediatamente disponibile.l pazienti con compromissione della funzione epatica o renale devono essere controllati frequentemente e il dosaggio deve essere attentamente adattato alla risposta del singolo paziente. In questi pazienti possono essere sufficienti dosaggio più bassi. Le stesse precauzioni valgono per pazienti anziani o debilitati e per pazienti con insufficienza respiratoria cronica. Come con tutti i farmaci depressori del SHC, l'uso di benzodiazepina puó precipitare una encefalopatia in pazienti con insufficienza epatica grave.Tavor Fiale non è raccomandato in pazienti con insufficienza epatica e/o renale condamata. Quando Tavor Fiale è usato in pazienti con patologia epatica o renale da lieve a moderata, la dose efficace più bassa deve essere considerata in quanto l'effetto del farmaco può essere prolungato. Cautela deve essere usata quando si somministra Tavor fiale ad un paziente con stato epilettico, specialmente se il paziente ha ricevuto altri farmaci depressori del sistema nervoso centrale o è gravemente malato. Deve essere considerata la possibilità che possa verificarsi arresto respiratorio o una parziale ostruzione delle vie aeree, e pertanto deve essere disponibile un'appropriata attrezzatura per la rianimazione.Studi dinici hanno mostrato che pazienti oltre i 50 anni possono avere una più profonda e prolungata sedazione con i lavor Fiale somministrato per via endovenosa. Normalmente una dose iniziale di $2 \mathrm{mg}$ è adeguata, a meno che non si desideri un grado maggiore di sedazione e/o amnesia. Uso pediatrico La sicurezza e l'efficacia di Tavor Fiale nei bambini non sono state stabilite e, pertanto, l'uso del prodotto è generalmente controindicato. 4.5 Interazioni medicamentose ed altre Si raccomanda di evitare l'assurcione contemporanea di alcool, poiché ciò può aumentare l'effetto sedativo del farmaco (vedere anche "Awrertenze").Le benzodiazepine, compreso Tavor Fiale, se associate ad altri farmaci ad attività depressiva centrale come barbiturici, antipsicotici, sedativi/ipnotici, ansiolitici, antidepressivi, analgesici narcotici, antistaminici sedativi, anticonvulsivanti e anestetici nnforzano gli effetti depressivi sul sistema nervoso centrale. Pertanto, è necessario o evitare del tutto queste sostanze o diminuirne le dosi.Quando la scopolamina è stata usata concomitantemente al Tayor Fiale, è stato osservato un aumento dell'incidenza di sedazione, allucinazioni e comportamento irrazionale.Sono stati niportati casi di eccessivo stupor, niduzione significativa della trequenza respiratoria $e$, in un caso, ipotensione, quando lorazepam è stato somministrato concomitantemente alla loxapina.Nel caso di assunzione concomitante di lorazepam e clozapina sono stati riportati sedazione marcata, eccessiva salivazione e atassia.|riemerone neuf mulsi do uBouronoCon l'uso di Tavor Fiale non sono state riportate o identificate interterenze nelle analisi di laboratorio. 4.6 Uso in caso di gravidanza e di allattamento le benzodiazepine non devono essere usate durante la gravidanza, specialmente nel primo e terzo trimestre. Se il farmaco è prescritto ad una donna in età feconda. essa deve essere awisata di contattare il proprio medico per l'interruzione del trattamento nel caso essa intenda intraprendere una gravidanza o sospetti di essere incintal'assunzione di benzodiazepine durante la gravidanza può causare danni al feto. Un aumentato rischio di malformazioni congenite associato con l'uso di agenti ansiolitici, quali il clordiazepossido, il diazepam ed il meprobamato, $\dot{e}$ stato suggerito in diversi sudi.Neli'vomo i livelli ematici, ottenuti dal cordone ombelicale, indicano che le benzodiazepine e i loro glucuronidi passano attraverso la placenta. E' stato riportato che neonati di madri che hanno assunto benzodiazepine per alcune settimane o più prima del parto hanno mostrato sintomi da sospensione durante il periodo postnatale. Sintomi quali ipoatrivita, ipotonia, ipotermia, depressione respiratoria, apnea, problemi di nutrizione e risposte metaboliche alterate da diminuita resistenza al freddo sono stati niportati in neonati le cui madri hanno fatto uso di benzodiazepine durante la fase tardiva della gravidanza o durante il parto.Sembra che nei neonati la coniugazione del lorazepam arvenga lentamente essendo il suo glucuronide rintracciabile nelle urine per più di 7 giorni. La glucuronizazione del lorazepam puó inibire competitivamente la coniugazione della bilirubina, portando alliperbilirubinemia nel neonato.Ci sono dati insufficienti riguardo la sicureza di Tavor parenterale in ostetricia, induso l'uso nel parto cesareo. Questo uso percio non è raccomandato.E' stato provato che il lorazepam è escreto nel latte umano in quantità farmacologicamente insignificante. Tavor fiale comunque, non deve essere somministrato durante l'allattemento. 4.7 Effetti sulla capacità di guidare e sull'uso di macchine I pazienti devono essere awvertiti di non guidare o intraprendere attività che richiedono una certa attenzione per 24-48 ore dopo la somministrazione di Tavor Fiale (vedere "Awertenze"). 4.8 Effetti indesiderati Gli effetti collaterali osservati più trequentemente con lavor Fiale rappresentano un'estensione degli effetti depressivi del farmaco sul sistema nervoso centrale. Sonnolenza eccessiva e torpore sono gli effetti collaterali principali. Le incidenze riportate dipendono dal dosaggio, dal modo di somministrazione, dall'uso concomitante di altri agenti depressivi del sistema nervoso centrale e dalle aspettative dellinvestigatore circa il grado e la durata della sedazione.La deambulazione prematura puó provocare cadute e conseguenti lesioni.Quando Tavor Fiale è somministrato per via endovenosa prima dell'anestesia locale o regionale, specialmente a dosaggi maggiori di $0.05 \mathrm{mg} / \mathrm{kg}$ o quando vengono somministrati agonisti oppiacei o agonisti pariali concomitantemente a dosaggi raccomandati di Tavor, può verificarsi sedazione eccessiva e torpore: questi efferti possono interferire con la collaborazione e la comunicazione del paziente.E' stato osservato che quando Tavor Fiale è stato dato per via endovenosa a pazienti di oltre 50 anni di età si è avuta un'incidenza più alta di sedazione eccessiva rispetto a pazienti con etrà inferiore ai 50 anni. Sono stati riportati irrequietezza, confusione, depressione, pianto, singhiozi, allucinazioni, senso di instabilità e diplopia. Ipertensione ed ipotensione sono stati osservati occasionalmente.Si sono verificate depressione respiratoria e ostruzione parziale delle vie aeree che hanno comportato una temporanea ipoventilazione. Rash cutaneo, nausea e vomito sono stati notati occasionalmente in pazienti che hanno ricevuto Tavor Fiale combinato con altri farmaci durante P'anestesia ed interventi chirurgici.Effetti locali - Dolore nel sito di iniezione, sensazione di bruciore e arrossamento nella stessa area sono stati riportati dopo somministrazione

intramuscolare di Tavor Fiale. Flebite locale, dolore immediatamente dopo liniezione $\mathrm{e}$ arrossamento nelle successive 24 ore sono stati niportati dopo somministrazione endovenosa di Tavor fiale.Reazioni paradosse, quali irrequieteza, agitazione, imitabilità, aggressività, delusione, rabbia, inabi, allucinazioni, psicosi e comportamento inappropriato, sono state occasionalmente riportate durante l'uso di benzodiazepine. E' piu probabile the queste rearioni si verifichino in bambini e anziani. Se questo si verificasse il trattamento deve essere interrotto. L'iniezione intra-arteriosa può provocare uno spasmo dell' arteria che può portare a gangrena, per la quale può essere richiesta amputazione (ved. "Controindicazioni"),4.9 Sovradosaggio Mel trattare liperdosaggio di qualsiasi farmaco è necessario tenere sempre presente che possono essere state prese diverse altre sostanze.siriomliperdosaggio delle benzodiazepine si manifesta di solito con depressione del sistema nervoso centrale di grado variabile dalla sonnolenza al coma. Nei casi leggeri i sintomi includono sonnolenza, confusione mentale e letargia. Nei casi più gravi, e specialmente quando sono stati ingeriti altri farmaci depressivi del sistema nervoso centrale o alcool, i sintomi possono comprendere atassia, ipotensione, ipotonia, depressione respiratoria, coma di $1^{\circ}-3^{\circ}$ grado e, molto raramente, morte.Tumaukrroll trattamento del sorradosaggio é principalmente di supporto fino a quando il farmaco è stato eliminato dall'organismo.I segni vitali e il bilancio idrico devono essere accuratamente tenuti sotto controllo. Un'adeguata funzionalità delle vie respiratorie deve essere mantenuta e usata la respirazione artificiale se necessario. Lipotensione, sebbene poco probabile, puó essere controllata con la noradrenalina. II lorazepam è scarsamente dializzabile.ll flumazenil, antagonista benzodiazepinico, può essere utile nei pazienti ospedalizzati per il trattamento delliperdosaggio con benzodiazepine. Prima di usario è necessario consultare le informazioni sul prodotto. 5. PROPRIETA FARMACOLOGICHE 5.IProprietà farmacodinamiche Lorazepam (Tavor), agente antiansia, è una 1.4 benzodiazepina avente il seguente nome chimico: 7-doro-5-(0-dorofenil)-1.3-diidro-3-idrossi-2H-1.4-benzodiazepin-2-one.Lorazepam è una polvere quasi bianca, pressoche insolubile in acqua e poco solubile in alcool e doroformio, avente punto di husione compreso to $166^{\circ} \mathrm{C}$ e $168^{\circ} \mathrm{C}$. II suo peso molecolare è $321.2 . \mathrm{La}$ sua formula di struttura è ha

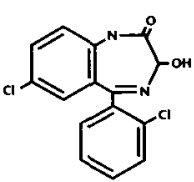

seguente: Ampi studi farmacologici e dinici hanno dimostrato che Javor Fiale possiede una azione ansiolitica, sedativa e anticonvulsivante. Tavor Fiale, utilizzato a dosi appropriate prima di un intervento chirurgico, procedure diagnostiche, 0 chemioterapia tumorale, riduce l'ansia, induce sedazione e riduce il ricordo di eventi associati. La mancanza di rievocazione e di riconoscimento oggettivo sono in genere ottimali entro 2 ore dalla somministrazione intramuscolare e entro 15 20 minuti dalla somministrazione endovenosal'esatto meccanismo di azione delle benzodiazepine non è stato ancora chiarito; comunque, sembra che le benzodiazepine agiscano attraverso vari meccanismi. Presumibilmente le benzodiazepine esercitano i loro effetti attraverso un legame con specifici recettori a diversi siti entro it sistema nervoso centrale, o potenziando gli effetti di inibizione sinaptica o presinaptica mediata dall'acido gamma-aminobutirico, oppure influenzando direttamente i meccanismi che generano il potenziale d'azione. 5.2 Proprietà farmacocinetiche lavor Fiale viene rapidamente assorbito quando somministrato per via intramuscolare. Le concentrazioni massime nel plasma vengono raggiunte entro 60-90 minuti circa dopo somministraxione intramuscolare. L'emivita di eliminazione di lorazepam non coniugato nel plasma umano è di circa 12-16 ore quando somministrato per via intramuscolare o endovenosa. Alle concentrazioni dinicamente significative, Javor si lega per il $90 \%$ circa alle proteine plasmatiche La coniugazione con acido glucuronico per formare il glucuronide inattivo rappresenta la via metabolica principale del Tavor Fiale. Non ci sono metaboliti attivi. II 70-75\% della dose viene escreto come gilucuronide nelle urine. Lorazepam non risulta idrossilato in modo significativo, né rappresenta un substrato per gli enzimi N-dealchilanti del sistema citocromico P450.L'età non ha alcun effetto dinicamente signiffcativo sulla cinetica del lorazepam. In uno studio è stata riportata, in soggetti anziani, una diminuzione statisticamente significativa nella dearance totale, ma l'emivita di eliminazione nonè stata significativamente alterata. Messuna alterazione nella dearance del lorazepam è stata riportata in pazienti con compromissione epatica da lieve a moderata (cioé epatite e cirrosi alcolica). Studi di farmacocinetica con dosi singole condotti in pazienti con insufficienza renale variante da lieve a grave non hanno riportato alcuna alterazione significativa nell'assorbimento, clearance o escrezione del lorazepam. L'eliminazione del gluaronide inattivo è risultata significativamente nidotta. Una ridotta eliminazione del lorazepam, associata ad un prolungamento dell'emivita, è stata riportata dopo somministrazione subcronica in uno studio su 2 pazienti con insufficienza renale cronica. L'emodialisi non ha avuto alcun effetto significativo sulla farmacocinetica del lorazepam come tale ma ha provocato una rimozione sostanziale del glucuronide inativo dal plasma. 5.3 Dati di tollerabilità preclinicaCarcinogenesi, mutagenesi e studi sulla fertilità Nessuna prova di potenziale carcinogenico è emersa in ratti o topi durante uno studio di 18 mesi con lorazepam somministrato per via orale. Un'indagine sull'attività mutagenica del lorazepam nella Drosophila melanogaster ha indicato che questo farmaco è inattivo dal punto di vista mutagenico.Uno studio sui ratti nel periodo precedente l'impianto dell'ovulo, effettuato con lorazepam per via orale alla dose di $20 \mathrm{mg} / \mathrm{kg}$, non ha evidenziato alcuna riduzione della fertilità. 6. INFORMAZIONI FARMACEUTICHE 6.I Lista degli eccipienti Glicole polietilenico 400 , alcool benzilico, glicole propilenico. 6.2 Incompatibilità Ved. "Interazioni" e "Posologia". 6.3 Durata di stabilità 2 anni. 6.4 Speciali precauzioni per la conservazione Proteggere dalla luce. Conservare in frigorifero $\left(0-4^{\circ} \mathrm{C}\right)$; non congelare. 6.5 Natura del contenitore, confezioni e relativi prezzi 5 fiale di vetro da $1 \mathrm{ml}$, contenenti ciascuna $4 \mathrm{mg}$ di lorazepam 6.6 Istruzioni per l'uso Vedere "Posologia e modo di somministrazione" 7. RAGIONE SOCIALE E DOMICILIO DEL TITOLARE A.I.C.WYETH S.p.A - Via Mettunense 90, Aprilia (Latina).8. NUMERO DI A.I.C. A.I.C. n. 022531139 9.1 DATA DELLA PRIMA AUTORIZZAZIONE/RINNOVO DELL'AUTORIZZAZIONE 9 Marzo 1995. 9.2 Eventuale tabella di appartenenza secondo il D.P.R. 9 ottobre 1990, n./ 309 Tabella V 9.3 Regime di dispensazione al pubblico Uso riservato agli ospedali e case di cura. Vietata la vendita al pubblico.10. DATA DI ULTIMA REVISIONE Gennaio 1997 


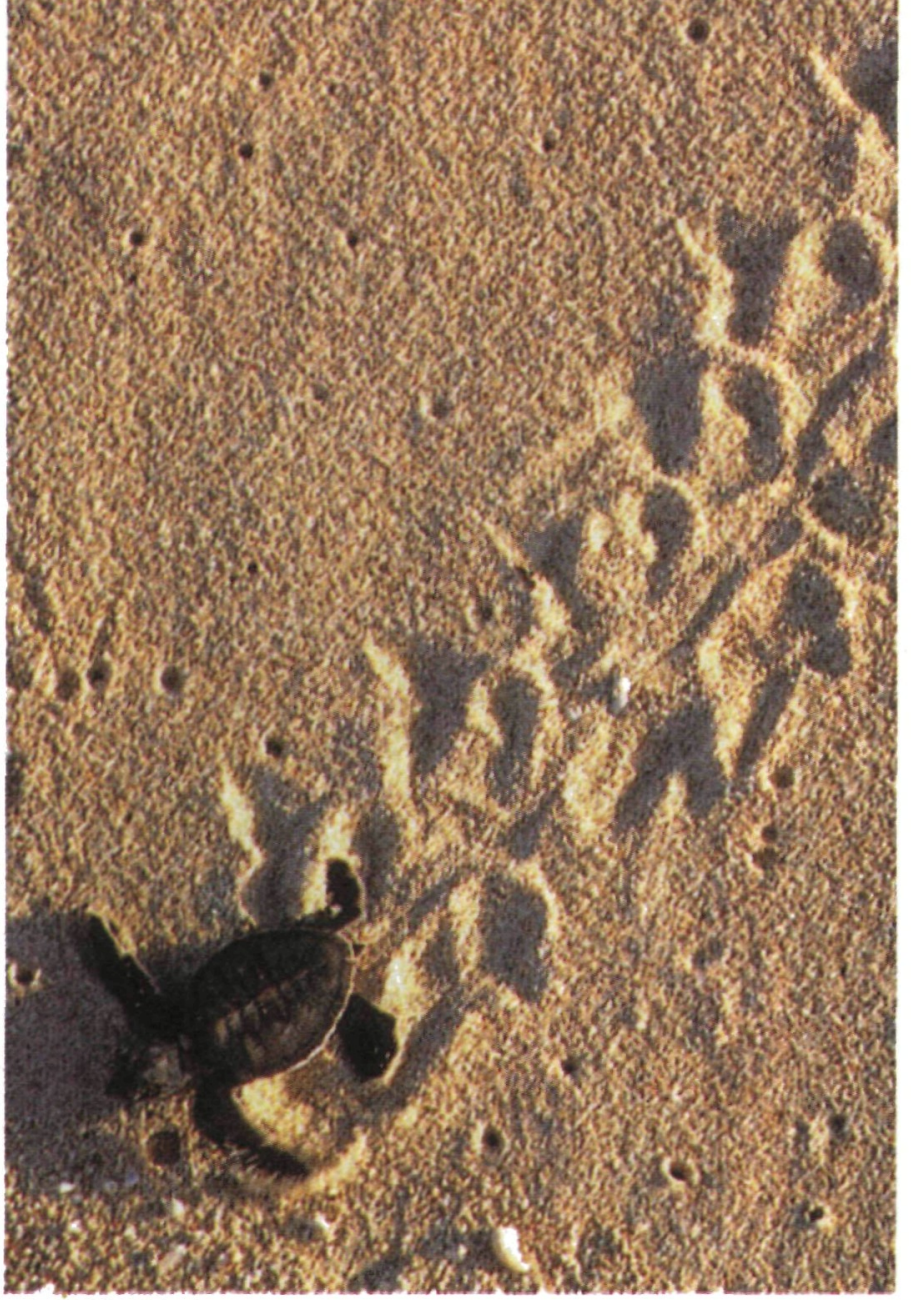

Numero Verde

$167-259620$

Questo è il Numero Verde

riservato ai nostri abbonati, nato per rispondere più

velocemente alle vostre

esigenze.
FERMOPOSTA

Il disservizio postale ha

raggiunto livelli non più giustificabili.

Il ritardo nella distribuzione

delle pubblicazioni periodiche è talvolta superiore ai due mesi e purtroppo la mancata o ritardata consegna delle riviste condiziona in misura determinante la decisione di rinnovare il proprio abbonamento.

Segnalateci ogni problema: sarà nostra cura rispedire copie non recapitate o giunte danneggiate. 


\section{EPIDEMIOLOCIA \\ EPSCCHIATRIA SOCIALE}

Volume 6, Numero 2, Maggio-Agosto 1997

\section{CONTENTS}

Guidelines for the preparation of $\mathrm{CV}$ and list of publications. To standardize the format for encouraging a more homogenous. evaluation of candidates for positions in psychiatry, M. Tansella, P. Pancheri, G.B. Cassano

\section{Editorials}

Psychotherapy research: why is it neglected in Italy? G.A. Fava

The efficacy of psychotherapy, J.C. Markowitz

Re-examining the hedgehogs anecdote, $R$. Carli

Psychotherapy research: linking efficacy to policy and practice, G. Parry, A.D. Roth

\section{Articles}

M. Chiappelli, F. Pileggi, G. Rosini, G. Neri, A three years follow-up of a group of «everyday patients» in a mental health centre

E. Guerriero, Psychiatric services, social co-operatives, voluntary organisations.

New relationship for new strategies

E. Favaretto, S. Torresani, The parental bonding as predictive factor for the development of adult psychiatric disorders

G. Fattore, M. Percudani, C. Pugnoli, A. Contini, Costs of psychiatric services: a study performed in a public mental center in Lombardy Region, Italy

Italian Society of Psychiatric Epidemiology (SIEP)

Third National Meeting of the SIEP (Milano 19th-21st October 1997) 


\section{SEROXII}
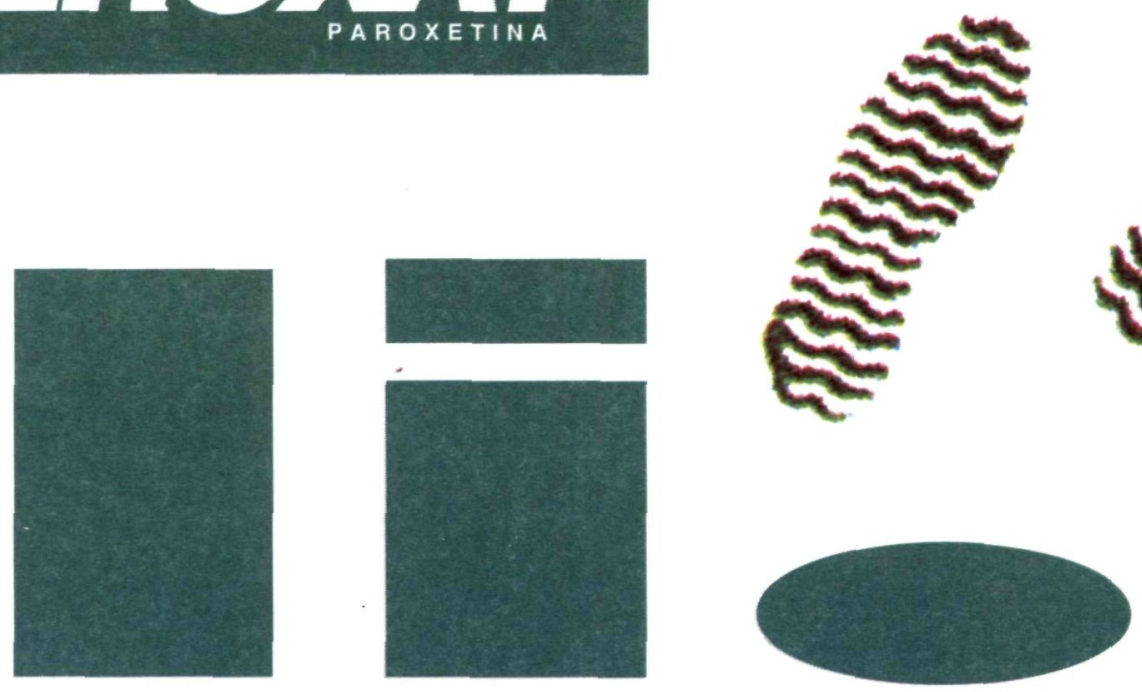

Indicazioni:

- Depressione di tutti i tipi, compresa la depressione accompagnata da ansietà.

- Prevenzione delle recidive e delle ricadute della depressione.

- Disturbo da attacchi di panico con o senza agorafobia.

- Disturbo ossessivo/compulsivo e prevenzione delle sue recidive.

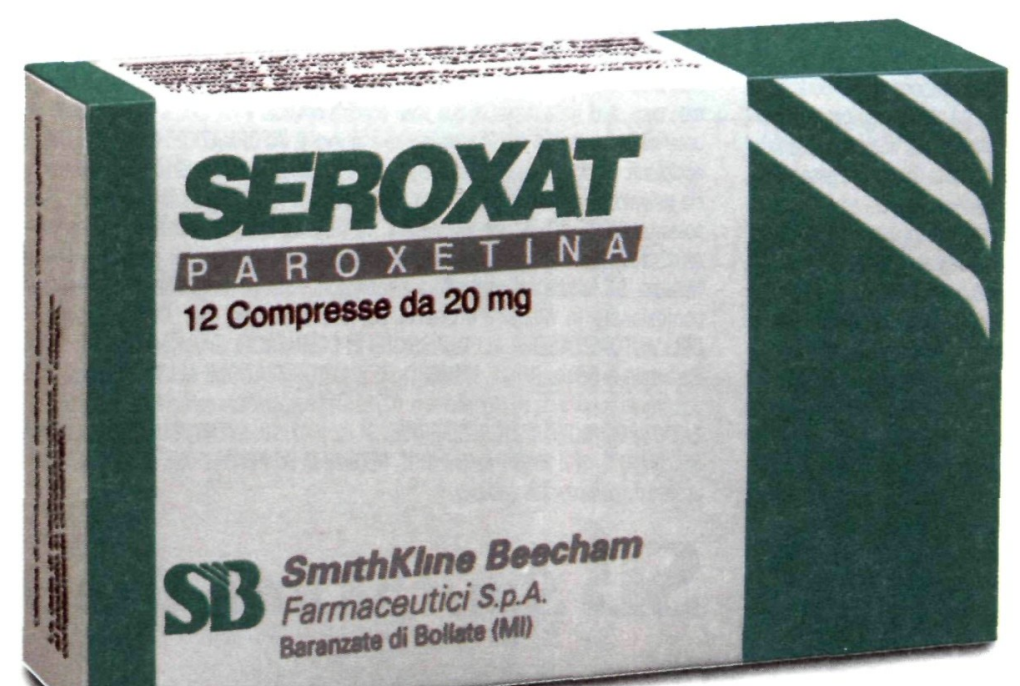




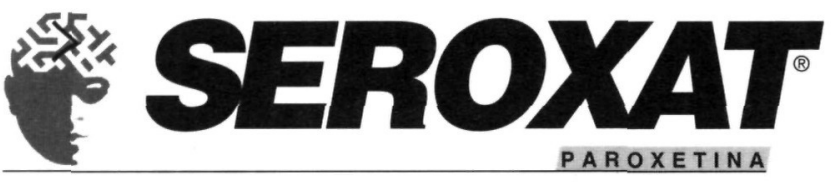

SCHEDA TECNICA

1. DENOMINAZIONE DELLA SPECIALITÀ MEDICINALE: SEROXAT. 2. COMPOSIZIONE QUALITATIVA E QUANTITATIVA IN PRINCIPI ATTIVI. Ogni compressa rivestita contiene: Principio attivo: Paroxetina cloridrato $22,88 \mathrm{mg}$ (corrispondente a paroxetina $20 \mathrm{mg}$ ) 3. FORMA FARMACEUTICA. Compresse rivestite divisibili, per uso orale. 4. INFORMAZIONI CLINICHE. 4.1 Indicazioni terapeutiche. Seroxat è indicato nel trattamento della depressione di tutti i tipi, compresa la depressione accompagnata da ansietà e nella prevenzione delle recidive e delle ricadute della depressione. Seroxat è indicato nel trattamento del disturbo ossessivo compulsivo e nella prevenzione delle sue recidive. Seroxat è indicato nel trattamento del disturbo da attacchi di panico con o senza agoratobia. L'aggiunta di paroxetina alla terapia cognitiva di tipo comportamentale si è dimostrata significativamente piu efficace nel trattamento del disturbo da attlacchi di panico rispetto alla terapia cognitiva comportamentale da sola. 4.2 Posologia e modo di somministrazione. Depressione. La dose raccomandata è di $20 \mathrm{mg}$, una volta al giorno. II dosaggio puo essere aumentato fino a $50 \mathrm{mg}$ al giorno, in base alla risposta del paziente, con aumenti graduali di $10 \mathrm{mg}$. Disturbo ossessivo compulsivo. La dose raccomandata è di $40 \mathrm{mg}$ al giorno. La dose iniziale è di $20 \mathrm{mg}$ al giorno e può essere aumentata settimanalmente con aument graduali di $10 \mathrm{mg}$ in base alla risposta del paziente. In alcuni casi il dosaggio puo essere aumentato fino ad un massimo di $60 \mathrm{mg}$ al giorno. Disturbo da attacchi di panico. La dose raccomandata è di $40 \mathrm{mg}$ al giorno. La dose iniziale e di $10 \mathrm{mg}$ al giorno e puo essere aumentata settimanalmente con aumenti graduali di $10 \mathrm{mg}$ in base alla risposta del paziente. Un basso dosaggio iniziale è raccomandato per ridurre al minimo il potenziale peggioramento della sintomatologia da panico, come si è osservato generalmente nel trattamento iniziale del disturbo di panico. Come per tutti i farmaci antidepressivi, il dosaggio, in base alla risposta terapeutica, deve essere aggiustato e rivisto se necessario entro le prime due o tre settimane dall inizio della terapia, e nel caso di interruzione del trattamento è opportuno che questo awenga gradualmente in quanto una interruzione repentina della somministrazione puo comportare la comparsa di sintomi quali disturbi del sonno, irritabilità, vertigini, sudorazione, nausea, disturbi sensoriali. Nell'anziano, visti i risultati degli studi farmacocinetici, è consigliabile un dosaggio di $20 \mathrm{mg}$ al giorno. In alcuni pazienti puo essere necessario aumentare la dose: questo deve comunque awenire gradualmente con incrementi di $10 \mathrm{mg}$ alla volta fino ad un massimo di $40 \mathrm{mg}$ a giorno in accordo alla risposta del paziente. In pazienti con insufficienza renale grave (clearance della creatinina $<30 \mathrm{~m} / \mathrm{min}$ ) 0 insufficienza epatica grave si verifica un aumento delle concentrazioni plasmatiche $\mathrm{d}$ paroxetina, pertanto si raccomanda la somministrazione del dosaggio minimo previsto. I pazienti dovrebbero essere trattati per un periodo sufficiente da assicurare la remissione completa dei sintomi, tale periodo può essere di molti mesi sia per la depressione, sia per il disturbo ossessivo compulsivo e per il disturbo da attacchi di panico. 4.3 Controindicazioni. Ipersensibilità verso i componenti o verso altre sostanze strettamente correlate dal punto di vista chimico. Non somministrare in età pediatrica. Generalmente controindicato in gravidanza e durante l'allattamento. 4.4 Speciali avvertenze e precauzioni per l'uso. Come nella maggior parte delle terapie con antidepressivi, Seroxat non dovrebbe essere somministrato in associazione con anti-MAO prima di due settimane dal termine di tale trattamento. Analogamente non è opportuno iniziare una terapia con ant-MAO prima di due settimane dal termine della terapia con paroxetina. Seroxat non produce variazioni cliniche significative della pressione arteriosa, della frequenza cardiaca e dell'ECG, ciononostante occorre osservare le consuete precauzioni in presenza di alterazioni cardiovascolari. Come nel caso di altri antidepressivi, Seroxat dovrebbe essere usato con cautela in pazienti epilettici. Complessivamente lincidenza di convut sioni in pazienti trattatic con Seroxat è stata $<0,1 \%$. II trattamento con Seroxat deve essere sospeso nei pazienti che presentino convulsioni. Seroxat dovrebbe essere usato con precauzione in pazienti con storia clinica di mania. Particolare attenzione occorre nell'uso di Seroxat nei pazienti in trattamento con anticoagulanti orali. Studi condotti con cimetidina hanno dimostrato che quest'uttima determina un aumento della biodisponibilità $\mathrm{di}$ paroxetina di circa il 45\%, pertanto si raccomanda l'impiego di Seroxat al dosaggio minimo. Esiste un'espe rienza clinica limitata nella somministrazione concomitante di paroxetina con ECT. Tenere fuori della portata dei bambini, 4.5 - Interazioni medicamentose e altre. Specifici studi sono stati condotti per valutare le possibili interazioni tra pâroxetina e farmaci o sostanze psicoattive. L'esperienza in un limitato numero di soggetti sani ha dimostratog che paroxetina non aumenta la sedazione e la debolezza associate a aloperidolo, amobarbital o oxazepam, quando somministrati contemporaneamente. Gli studi condotti nell'animale indicano che può verificarsi una interazione tra paroxetina e inibitori delle monoaminoossidasi (anti-MAO) o triptofano, analogamente ad altri inibitori della ricaptazione di serotonina, e che puo determinare in tali circostanze la "sindrome da serotonina" caratterizzata da agitazione, irequiletezza e sintomi gastrointestinali quali diarrea. In uno studio in pazienti depressi stabilizzati con siflo nors s e osservata interazione farmacocinetica tra paroxetina e litio. Dato che lesperienza clinica è limitata, occorte particolare attenzione nella somministrazione contemporanea di paroxetina e litio. La co-somministrazione di paroxetina e fenitoina è associata ad una diminuzione nella concentrazione plasmatica drparoxetina. Quando questi due farmaci sono co-somministrati non è necessario un aggiustamento della dose iniziale con paroxetina, ogni eventuale aggiustamento della dose dovrà essere valut tato sulla base della risposta clinica. L'assunzione con anticonvulsivanti può essere associata ad un aumento della incidenza di reazioni indesiderate. Sebbene la paroxetina non aumenti gli effetti dannosi psicomotori indotti dalla assunzione di alcool, il loro uso concomitante è sconsigliato. Come con altri antidepressivi, inclusi gli SSRI, Seroxatouo, a livello del citocromo P 450, interferire sul metabolismo epatico di alcuni farmaci dete minandone laumento dei livelli plasthatici, fra questi debrisochina, sparteina, alcuni antidepressivi triciclici neuroletticé, fenotiazhici e gl antiarthmici di classe 1 C. II metabolismo e la farmacocinetica di Seroxat possono essere influenzati dai farmaç che provocano una induzione o un'inibizione del metabolismo enzimatico. Nel caso in cul Seroxat sia somministrato contemporaneamente a farmaci inibitori del metabolismo enzimatico si suggerisce l'uso dei dosaggi piü bassi. Nel caso di co-somministrazione contemporanea a farmacinduttori de metabolismo enzimatico, non è richiesto alcun aggiustamento iniziale del dosaggio. Ogni successivo aggiustamento deve essere basato sulla risposta clinica. Una interazione larmacocinetica sighiticativa e stata eviden-

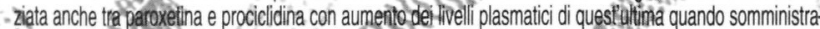
" te contemporaneamente. Se si ossenvano effeffianticolinergici la dose di procieidina dovrebbe essere ridotta. "Dati preliminari suggeriscono una possibile interazione farmacodinamica tra paroxetina e waffarina che puo compotare un allungamento del tempo di emorragia in presenza di valori normali di protrombina. 4.6 Uso in caso di gravidanza e di allattamento. Nonostante gli studi negl animallichon abbiano dimostrato a icun effetto teratogeno o embriotossico selettivo, la sicurezza della paroxetinanentla conna in gravidanza son estata stabi lita; pertanto il prodotto non dovrebbe essere utilizzato durante laggravidanza o l'allattamento se non nei casi in cui i potenziale beneficio superi il possibile rischio e comunque solto il diretto controth der medico. 4.7 Effetti sulla capacità di guidare e sull'uso di macchine. L'espertenza clinica ha dimtostrato che la terapia con paroxetina non è associata ad alterazioni delle funzioni cogntuve o psicomotorie. Tuttavia, come con tutti i farmaci psicoattivi, i pazienti dovrebbero essere avvertiti di usare cautela nell'uso di macchinari pericolosi e nella guida di autoveicoli. 4.8 Effetti indesiderati. Le reazioni indesiderate con paroxetina sono di moderata entità e non influenzano la qualita di vita del paziente, generalmente non richiedono interruzione della terapia e durante il trattamento prolungato possono diminuire di intensità e frequenza. Le reazioni avverse associate all'uso di paroxetina piu comunemente osservate sono: nausea, sonnolenza, sudorazione, tremore, astenia, secchezza delle fauci, insonnia e disfunzioni sessuali, vertigini, costipazione, diarrea e diminuzione dell'appeti to. Raramente sono state riscontrate convulsioni. Occasionalmente sono stati riportati disturbi extrapiramicali (distonia oro-facciale) in pazienti con pregressi disturbi del movimento o in pazienti in trattamento con neurolettici, prevalentemente nell'anziano è stata riscontrata iponatriemia, che generalmente scompare con la sospensione del farmaco. Sono stati riscontrati raramente transitori aumenti degli enzimi epatici. Sebbene non sia stata dimostrata una relazione causale con paroxetina, si consiglia di sospendere il trattamento in caso d comparsa di alterazioni della funzionalità epatica. Paroxetina, rispetto agli antidepressivi triciclici, è associata ad una minore probabilità di causare secchezza delle fauci, costipazione e sonnolenza. 4.9 Sovradosaggio (sintomi, soccorsi d'urgenza, antidoti). I sintomi di sovradosaggio con paroxetina includono nausea, vomito, tremore, midriasi, secchezza delle fauci e irritabilità. Non si sono osservati casi di anormalità nell'ECG, coma o convulsioni successive al sovradosaggio con paroxetina. Casi di sovradosaggio sono stati segnalati con paroxetina fino a $2000 \mathrm{mg}$, da soli o in associazione con altri farmaci. Quando usato da solo, Seroxat non ha mai causato morte; il recupero del paziente non ha lasciato sequele. II trattamento si basa sulle abituali misure utilizzate nel sovradosaggio con antidepressivi: si consiglia svuotamento gastrico attraverso induzione di emesi o lavanda gastrica. Successivamente può essere somministrato carbone attivo, 20 o $30 \mathrm{~g}$ ogni $4-6$ ore nelle prime 24 ore dopo ingestione. Ė indicata una terapia di supporto con attenta osservazione e frequente monitoraggio dei sintomi vitali. La precoce somministrazione di carbone attivo può ritardare l'assorbimento d SerOXat. 5. PROPRIETA FARMACOLOGICHE E TOSSICOLOGICHE ED ELEMENTI DI FARMACOCINETF-

CA. 5.1 Farmacodinamica. La paroxetina è una molecola con una azione inibitoria potente e selettiva sulla ricaptazione della serotonina (5-didrossitriptamina: $5 \mathrm{HT}$ ) nei neuroni cerebrali, senza interferenze sulla captazione della noradrenalina. La sua efficacia nei trattamenti della depressione, del disturbo ossessivo compulsivo e del disturbo da attacchi di panico è presumibilmente correlata a tale meccanismo. Chimicamente è una ()-trans-4-(4'fluorotenil)-3-(3', ', metilendiossi-fenossimetil)-piperidina cloridrato, la cui struttura nor è riconducibile a quella degli antidepressivi triciclici, tetraciclici e di altri disponibili. Negli studi a lungo termine con paroxetina è emerso che l'efficacia si mantiene per periodi di almeno un anno nel trattamento della depressione e del disturbo ossessivo comoulsivo e per oltre un anno nel trattamento del disturbo da attacchi di panico. 5.2 Farmacocinetica. La paroxetina e ben assorbita nel tratto gastrointestinale dopo somministrazione orale. I livelli sistemici di equilibrio sono raggiunti entro 7-14 giorni dall 'inizio del trattamento. Circa il 95\% della paroxetina presente nel plasma è legato alle proteine. L'emivita di eliminazione e generalmente di un giorno. La paroxetina è metabolizzata a livello epatico; gli studi di farmacologia hanno evidenziato che i metabolit sono molto meno potenti della paroxetina, pertanto si presume che non contribuiscano al suo effetto terapeutico. I principali metaboliti sono prodotti polari e coniugati, prontamente eliminati. L'escrezione urinaria di paroxetina e circa il $64 \%$ della dose di cui meno de $2 \%$ in forma immodificata. Circa il $36 \%$ della dose e escre to nelle feci, attraverso la bile, di cui la forma immodificata rappresenta meno dell' $1 \%$. II profilo farmacocinetico non sembra modificarsi durante la terapia a lungo termine. La biodisponibilità della paroxetina non è influenzz ta dalla contemporanea assunzione di cibo. Nei soggetti anziani le concentrazioni plasmatiche di paroxetina sono risultate elevate. 5.3 Dati preclinici di sicurezza. Gli studi di tossicità acuta hanno evidenziato valori di LDso Dari a 374 e 341 makg dopo somministrazione orale e valori pari a 27 e $38 \mathrm{mg} / \mathrm{kg}$ dopo somministrazione e.v. rispettivamente nel ratto e nel topo. Negli studi di tossicità per somministrazioni ripetute nel ratto è stato evidenziato un lieve incremento dei valori di tosf́atasi alcalina a dosi pari a 4 molkg/die e di alaninaminotransterasi alle dosi di 12 e $40 \mathrm{mg} / \mathrm{kg} / \mathrm{die}$. Come atteso per le ammine lipofile, inclusi gli antidepressivi triciclici, è stata riscontrata fosfolipidosi nel ratto, ma non è stata osservata invece negli studi condotti nei primati della durata di un anno a dosi superiori a 6 volte il dosaggio clinico raccomandato. Nella scimmia Rhesus è stato evidenziato un aumento dei lisosomi nel fegato a dosi elevate dopo 12 mesi di trattamento. In questa specie la dose che non ha causato effetti tossici è stata di $1 \mathrm{mg} / \mathrm{kg} / \mathrm{die}$. Non è stata evidenziata teratogenicità nel ratto $\mathrm{e}$ nel coniglio alle dosi tossiche materne, mentre una embriotossicità non selettiva viene segnalata agli stessi livelli di dose. Nel ratto gli studi sulla fertilità hanno evidenziato alla dose di $13 \mathrm{mg} / \mathrm{kg}$ una frequenza ridotta delle gravidanze e, a $43 \mathrm{mg} / \mathrm{kg} / \mathrm{die}$ una minore sopravivenza post-partum dei nati. Dosi pari a $50 \mathrm{mg} / \mathrm{kg} / \mathrm{die}$ hanno causato effetti tossici sul ciclo estrale e sull'ovulazione; nei maschi invece sono state evidenziate variazioni istopatologiche a carico della rete testis e dell'epididimo. Nessun potenziale cancerogeno è stato riscontrato negli studi della durata di due anni, condotti nel ratto e nel topo e nessun effetto genotossico è stato osservato negli studi di mutagenesi in vitro e in vivo. 6. INFORMAZIONI FARMACEUTICHE. 6.1 Lista degli eccipienti. Calcio fosfato bibasico biidrato, idrossipropilmetilcellulosa 2910, sodio carbossimetilamido, magne sio stearato, idrossipropilmetilcellulosa, titanio biossido (E 171), polietilenglicole 400, polietilenglicole 6000 , polisorbato 80 (E 433). 6.2 Incompatibilità. Nessuna. 6.3 Durata di stabilità a confezionamento integro. 36 mesi a confezionamento integro, correttamente conservato. 6.4 Speciali precauzioni per la conservazione. Nessuna. 6.5 Natura del contenitore e confezioni. Astuccio contenente 12 compresse rivestite da $20 \mathrm{mg}$ confezionate in blister PVC/alluminio. 6.6 Istruzioni per l'uso. Nessuna. 7. TITOLARE DELL'AUTORIZZAZIONE ALL'IMMISSIONE IN COMMERCIO. SmithKline Beecham S.p.A. Via Zambeletti Baranzate di Bollate (MI). 8. NUMERO DELL'AUTORIZZAZIONE ALL'IMMISSIONE IN COMMERCIO. 12 compresse rivestite da 20 ing: codice n. 027963014 del Ministero della Sanità. 9. DATA DI PRIMA AUTORIZ. ZAZIONE/RINNOVO AUTORIZZAZIONE. 31.12.1992. 10. EVENTUALE TABELLA DI APPARTENENZA SECONDO IL DPR 309/90:- Nessuna. 11. REGIME DI DISPENSAZIONE AL PUBBLICO. Vendita su presentazione di ricettla medica ripetibile.

\section{S13 SmithKlıne Beecham}




\section{EPIDEMIOLOCIA EPSICHIATRIA SOCIALE}

\section{NORME PER GLI AUTORI}

Epidemiologia e Psichiatria Sociale (EPS) pubblica ricerche nel campo della salute mentale che utilizzano un approccio epidemiologico e ricerche di psichiatria sociale. Ė aperta a contributi di epidemiologi, psichiatri, psicologi, statistici ed altri ricercatori e di altri operatori dei servizi di salute mentale. E una rivista quadrimestrale (esce a marzo, luglio e novembre).

Oltre ad articoli in extenso e comunicazioni brevi (1500-2500 parole) che riportano i risultati di studi empirici potranno essere presi in considerazione contributi metodologici, revisioni della letteratura ed anche resoconti di esperienze pratiche di organizzazione o di trasformazione dei servizi per la salute mentale, purché adeguatamente documentati. I contributi dovranno essere in italiano $o$ in inglese. EPS pubblica anche Editoriali sia in inglese che in italiano, preparati su invito, che hanno lo scopo di mettere a fuoco lo stato di un problema e di esaminarne le prospettive.

Infine EPS pubblica Documenti, cioè informazioni e notizie su temi di assistenza psichiatrica, su aspetti legislativi e normativi, ecc., di interesse non solo per gli operatori ma anche per amministratori, tecnici e funzionari delle Aziende ULSS, delle Aziende Ospedaliere e delle Regioni e Strumenti, cioè scale, questionari ed altri strumenti di valutazione e di misura utili per la ricerca epidemiologica in psichiatria. I contributi completi di eventuali tabelle e figure, dovranno essere inviati, in 3 copie, al Direttore della Rivista, al seguente indirizzo:

Professor Michele Tansella, Servizio di Psicologia Medica, Istituto di Psichiatria, Ospedale Policlinico, 37134 Verona

L'accettazione dei contributi è subordinata all'approvazione da parte dei referee. L'invio di un contributo per una eventuale pubblicazione implica che si tratta di un lavoro inedito, non inviato per la pubblicazione altrove e la sua accettazione implica che non sarà successivamente pubblicato altrove.

\section{DATTILOSCRITTI}

I contributi dovranno essere dattiloscritti in doppia spaziatura, su di una sola faccia del foglio, lasciando ampi margini.

Nella prima pagina dovranno essere indicati: titolo del lavoro (in italiano ed in inglese), titolo abbreviato, nome (per esteso) e cognome degli autori, istituzione nella quale il lavoro è stato eseguito e parole chiave (in italiano ed in inglese); inoltre, a pie' di pagina, indirizzo completo dell'autore al quale vanno inviate la corrispondenza e le richieste di estratti. Allegare anche numero di Fax ed eventuale indirizzo E-mail. La seconda e la terza pagina dovranno contenere un riassunto rispettivamente in italiano e in inglese. Il riassunto ed il summary degli articoli in extenso e delle comunicazioni che riportano risultati di studi empirici dovranno avere una lunghezza maggiore degli altri (250-300 parole) e dovranno essere strutturati in paragrafi che sintetizzino il lavoro eseguito. I paragrafi dovranno essere intitolati: Scopo/Objective (sintetizzare lo scopo e gli obiettivi dello studio); Disegno/Design; Setting/Setting; Principali misure utilizzate/Main outcome measures; Risultati/Results (riassumere solo i risultati principali ottenuti) e Conclusioni/Conclusions (direttamente supportate dai dati).

Anche il riassunto ed il summary delle Revisioni della letteratura dovranno avere una lunghezza di $250-300$ parole; essi dovranno essere strutturati nei seguenti paragrafi:

Scopo/Objective (lo scopo principale della review), Metodo/Method (fonti consultate, criteri di inclusione e di esclusione degli studi che sono stati revisionati), Risultati/Results (principali risultati ottenuti) e Conclusioni/Conclusions (includere la rilevanza clinica o altre implicazioni che la revisione della letteratura può avere).

Tutti gli altri contributi devono avere riassunti e summaries standard, di non più di 150 parole.

Per gli Editoriali ed i contributi pubblicati nelle Sezioni denominate Documenti e Strumenti non è necessario il riassunto.

Eventuali ringraziamenti dovranno comparire alla fine del lavoro, prima della bibliografia.

Gli Autori devono osservare scrupolosamente le seguenti norme:

- dividere il dattiloscritto in paragrafi (ad es., per i lavori sperimentali: Introduzione, Metodi, Risultati, Discussione);

- sottolineare nel testo le parole e le frasi che dovranno essere stampate in corsivo;

- usare prima delle cifre decimali il punto, anziché la virgola (sistema anglosassone), sia nel testo, sia nelle tabelle e nelle figure.

- citare nel testo solo autori $i$ cui lavori vengano inclusi nella bibliografia. Per i lavori di un solo autore, citare cognome e anno della pubblicazione. Per i lavori pubblicati da due autori citare nel testo entrambi i cognomi, separati dal simbolo \& e seguiti dall'anno della pubblicazione. Per i lavori con più di due autori citare nel testo il cognome del primo, seguito da et al. e dall'anno della pubblicazione.

\section{BIBLIOGRAFIA}

Alla fine del contributo includere una lista bibliografica completa (preparata secondo il sistema Harvard). Seguire le seguenti istruzioni:

Articoli su riviste. Gli autori vanno citati tutti. Indicare cognome, iniziale del(i) nome(i) di ciascun autore, anno della pubblicazione (tra parentesi), titolo del lavoro (iniziale maiuscola solo per la prima parola del titolo), nome della rivista (per esteso e sottolineato) numero del volume, pagina iniziale e finale.

Bridges K., Goldberg D., Evans B. \& Sharpe T. (1991). Determinants of somatization in primary care. Psychological Medicine $21,473-483$

Articoli su Supplementi di riviste. Dopo gli autori, l'anno di pubblicazione e il titolo (vedi sopra) indicare titolo del supplemento (sottolineato; iniziale maiuscola per tutte le parole del titolo), iniziale del nome e cognome degli editors, nome della rivista (per esteso) e numero del supplemento, numero del volume, pagina iniziale e finale (precedute dal simbolo pp.).

Martini P., Cecchini M., Corlito G., D'Arco A. \& Nascimbeni P. (1985). A model of a single comprehensive mental health service for a catchment area: a community alternative to hospitalization. In Focus on the Italian Psychiatric Reform (ed. C. Perris and D. Kemali), Acta Psychiatrica Scandinavica, Supplementum No. 316, vol. 71, pp. 95-120.

Capitoli di libri. Dopo gli autori, l'anno di pubblicazione ed il titolo (vedi sopra) indicare titolo del libro (sottolineato; iniziale maiuscola per tutte le parole del titolo), iniziale del nome e cognome degli autori o degli editors, pagina iniziale e finale (vedi sopra), casa editrice, e dopo i due punti, luogo di pubblicazione.

Klerman G. \& Weissman M. (1989). Continuities and discontinuities in anxiety disorders. In The Scope of Epidemiological Psy- 


\section{NORME PER GLI AUTORI (segue)}

Articoli in Atti di Convegni, Seminari, ecc.

Balestrieri M., Arreghini E., Marino S. \& Bellantuono C. (1989). I disturbi emotivi nella medicina di base: una rassegna degli studi epidemiologici nell'area di Verona. In Atti del $37^{\circ}$ Congresso Nazionale della Società Italiana di Psichiatria, pp. 41-46. CIC Edizioni Internazionali: Roma.

Libri e Monografie.

Autore(i) individuale(i): ford.

Shepherd M., Cooper B., Brown A. C. \& Kalton G. (1966). Psychiatric Illness in General Practice. Oxford University Press: Ox-

Editor(s) o curatore(i):

Cooper B. (ed.) (1987). Psychiatric Epidemiology. Progress and Prospects. Croom Helm: London.

Autore istituzionale:

American Psychiatric Association (1987). Diagnostic and Statistical Manual of Mental Disorders (3rd. ed. revised). American Psychiatric Association: Washington DC.

\section{TABELLE E FIGURE}

Indicare nel testo dove vanno inserite eventuali tabelle e figure. Dattiloscrivere le tabelle su fogli separati, ciascuna su di un foglio. Le tabelle vanno numerate progressivamente con numeri romani e corredate di un titolo esaurientemente esplicativo.

Nel testo vanno citate senza abbreviazioni (es.: tabella I).

Allegare eventuali figure pronte per la riproduzione. Nel testo vanno citate senza abbreviazioni. Tutte le figure (grafici, disegni sche$\mathrm{mi}$, ecc.) devono essere contraddistinte con numeri arabi progressivi (es.: figura 1). Ogni figura dovrà essere corredata da una didascalia. Sia le figure che le didascalie vanno separate dal testo e fornite a parte. Sul retro di ogni figura indicare il nome del primo autore del contributo. Includere solo le tabelle e le figure ritenute essenziali.

\section{LINEE GUIDA PER L'INCLUSIONE DEI NOMI DEI COLLABORATORI TRA GLI AUTORI}

Nel caso di articoli firmati da più autori, ciascun autore deve aver partecipato al lavoro in misura sufficiente ad assumere pubblica responsabilità per il suo contenuto. Questa partecipazione deve includere: a) concepimento o disegno dello studio, oppure analisi e interpretazioni dei dati, o entrambe le cose; b) stesura dell'articolo o revisione critica del suo contenuto; c) approvazione finale della versione da pubblicare. Aver partecipato solo alla raccolta dei dati non giustifica il fatto di essere incluso tra gli autori. Tutti gli elementi (compresi nei punti a, b, c), che siano di importanza decisiva per le conclusioni del lavoro inviato per la pubblicazione, devono poter essere attribuiti ad almeno uno degli autori.

N.B. All'Autore indicato nella prima pagina, dopo l'accettazione del lavoro, verrà restituito il dattiloscritto con le correzioni e le modifiche editoriali del Redattore-Capo. Egli dovrà apportarle e rispedire al Dr. R. Fianco (Servizio di Psicologia Medica, Ospedale Policlinico, 37134 Verona) la versione finale del dattiloscritto (in due copie) più una versione su dischetto, sia in ASCII che nel Wordprocessor utilizzato (vedi sotto). Modifiche e variazioni eseguite dall'Autore in modo non preciso potranno ritardare la pubblicazione del lavoro.

L'Autore indicato nella prima pagina riceverà le bozze da correggere. Le correzioni dovranno limitarsi agli errori tipografici. Saranno forniti, per ogni articolo, 15 estratti gratuiti. Estratti supplementari potranno essere ordinati all'atto della correzione delle bozze.

\section{DISKETTE SUBMISSION INSTRUCTIONS}

Authors are requested to deliver the final, accepted version of their manuscripts on diskette.

- Storage medium. 3.5 inch diskette in Windows format.

- Software. Word 2.0 or 6.0 is preferred. The use of desktop publishing software (Aldus Pagemaker ${ }^{\circledR}$, Quark Xpress ${ }^{\circledR}$, etc.) is discouraged. If you prepared your manuscript with such a program, export the text to a wordprocessing format.

- Format. Keep the document as simple as possible. Refrain from any complex formatting. Do not use the footnote function of your word processor.

- Illustrations. Submission of electronic illustrations is encouraged, but not required. Submit illustrations on a separate diskette from the text. TIFF and EPS files or native application files are acceptable. For grey scale and color figure submissions please contact us for more detailed instructions.

- File name. Submit each article as a single file. Name each file with your last name (not to exceed 8 letters), followed by a period, plus the three-letter extension. If your last name exceeds eight letters, it should be trouncated to fit: For example, files prepared by author Abbagnale would be named: Abbagnal.EPS.

- Disk label. Label all diskettes with your name, the file name, and the program used.

- Paper copy. The diskette must be accompanied by hard copy printout. If the disk and paper copy differ, the paper copy will be considered the definitive version.

Please refer any questions to:

Dr. Renato Fianco

renatof@borgoroma.univr.it

Telephone: $+39-(0) 45-807.4441$

Fax: $+39-(0) 45-585.871$. 


\section{NOTIZIE AMMINISTRATIVE}

\section{Abbonamenti per l'anno 1997}

per l'Italia

Privati

Istituti, Enti, Biblioteche

Ospedali, USL

L. 75.000

er l'estero

L'abbonamento decorre da gennaio a dicembre.

In caso di disdetta dell'abbonamento è gradita una tempestiva comunicazione scritta.

Per i fascicoli eventualmente non ricevuti l'abbonato potrà fare richiesta all'editore. Se la richiesta sarà tempestiva, il fascicolo duplicato sarà fornito gratuitamente; altrimenti verrà addebitato in contro assegno (prezzo del fascicolo separato più spese postali).

Un fascicolo separato

L. 32.000

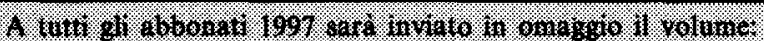

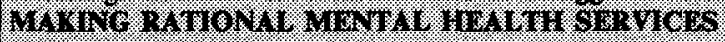

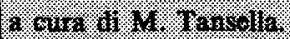

Questo periodico è iscritto all'U.S.P.I. Unione Stampa Periodica Italiana

\section{Pagamento}

Per abbonamenti arretrati e fascicoli arretrati sono validi i prezzi dell'anno corrente.

Le richieste e i versamenti debbono essere effettuati a:

Il Pensiero Scientifico Editore - Ufficio Abbonamenti - via Bradano, 3/C - 00199 Roma, tel. (06) 86207158 - 86207159 86207168 - 86207169 - fax (06) 86207160.

La quota di abbonamento può essere pagata a mezzo di assegno circolare, assegno di conto corrente, vaglia postale. Può anche essere versata sul c/c postale n. 902015.

Gli Enti, Istituiti, Biblioteche, Ospedali, USL, che desiderano la fattura dovranno farne richiesta al momento dell'ordine di abbonamento. Questo sarà attivato dopo il saldo della fattura.

Non saranno evase richieste di ricevute o fatture successive al momento dell'ordinativo.

L'I.V.A. è compresa nel prezzo di abbonamento (art. 74/C D.P.R. 633/72).

\section{Inserzioni pubblicitarie}

Le richieste vanno indirizzate a: Il Pensiero Scientifico Editore Servizi di Comunicazione Integrata

Via Bradano 3/c - 00199 Roma

Tel. 06/86207165 (diretto). Fax 06/86207160.

E-mail: pensiero@pensiero.it

Internet: http://www.pensiero.it 


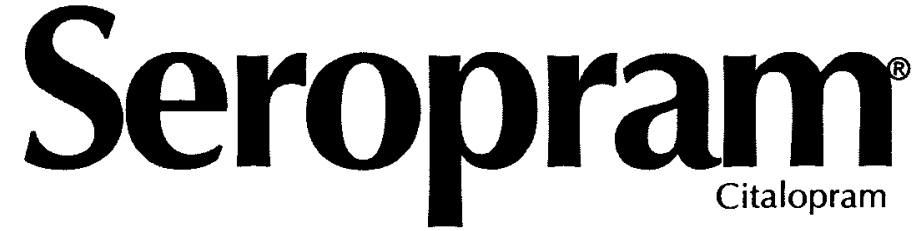

Riassunto delle caratteristiche del prodotto 1. DENOMINAZIONE DELLA SPECIALITA. Seropram 20

2. COMPOSIZIONE QUALI-QUANTITATIVA.

Una compressa da $20 \mathrm{mg}$ contiene: Principio attivo

Citalopram bromidrato

pari a citalopram

Eccipienti

Amido di mais

Lattosio

Cellulosa microcristallina

Polivinilpirrolidone vinilacetato

Glicerina

Sodio croscarmellosio

Magnesio stearato

Titanio biossido

Idrossipropilmetilcellulosa

Polietilenglicole 400

3. FORMA FARMACEUTICA.

$24,98 \mathrm{mg}$
$20,00 \mathrm{mg}$

$46,10 \mathrm{mg}$

$23,10 \mathrm{mg}$

$18,80 \mathrm{mg}$

$06,25 \mathrm{mg}$

$02,50 \mathrm{mg}$

$02,50 \mathrm{mg}$

$\infty, 70 \mathrm{mg}$

$02,00 \mathrm{mg}$

00,40

\section{Compresse rivestite.}

4. PROPRIETA FARMACOLOGICHE TOSSICOLOGICHE E FARMACOCINETICHE.

Citalopram è un nuovo derivato biciclico ftalenico con effetto antidepressivo. Studi biochimici e comportamentali hanno dimostrato che l'effetto farmacodinamico del citalopram è strettamente correlato a una potente inibizione dell'uptake della 5-HT (5-idrossitriptamina = serotonina). Citalopram non ha effetto sull'uptake della NA (noradrenalina) ed è quindi l'inibitore più selettivo dell'uptake della serotonina finora descritto, come dimostrato dal rapporto pari a 5000 tra le concentrazioni inibenti l'uptake della NA e delia serotonina. Non presenta alcuna influenza sull'uptake della DA (dopamina) o del GABA (acido gamma-aminobutirrico). Inoltre, né citalopram, né i suol metaboliti hanno proprietà antidopaminergiche, antiadrenergiche, antiserotoninergiche, antiistaminergiche o anticolinergiche e non inibiscono le MAO (monoaminoossidasi). Citalopram non si lega ai recettori delle benzodiaze pine, del GABA o degli oppioidi. Dopo trattamento prolungato, l'efficacia inibitoria sull'uptake della 5-HT è invariata; inoltre citalopram non induce variazioni nella densità dei neurorecettori come accade con la maggio parte degli antidepressivi triciclici e con i più recenti antidepressivi atipici Sono assenti gli effetti sui recettori colinergici muscarinici, sui recettori istaminici e sugli alfa-adrenorecettori, con conseguente mancata insorgenza degli effetti collaterali legati alla inibizione di questi recettori: secchezza delle fauci, sedazione, ipotensione ortostatica, presenti dopo trattamento con molti farmaci antidepressivi. Citalopram è quindi unico per la sua estrema selettività di blocco dell'uptake e per l'assenza di attività agonista 0 antagonista sui recettori. II farmaco non ha potere teratogeno e non influisce sulla riproduzione o sulle condizioni perinatali, non ha effetto mutageno né carcinogenetico. Citalopram è rapidamente assorbito dopo somministrazione orale. I livelli plasmatici massimi di citalopram sono raggiunti entro 2-4 ore dalla somministrazione. II volume apparente di distribuzione è circa 14 $1 / \mathrm{kg}$ (range 12-16 l/kg). II legame alle proteine plasmatiche è inferiore all' $80 \%$. La biodisponibilità di citalopram dopo somministrazione orale è praticamente completa. È stata dimostrata una relazione lineare tra le concentrazioni plasmatiche allo stato stazionario e la dose somministrata, con concentrazioni medie di circa $250 \mathrm{nM}$ per una dose giornaliera pari a $40 \mathrm{mg}$ Lemivita biologica è di circa un giorno e mezzo e nella maggior parte de pazienti lo stato stazionario è raggiunto entro la prima settimana di terapia. Nella maggior parte dei pazienti i livelli dello stato stazionario sono compresi nel range 100-400 nM per una dose giornaliera pari a $40 \mathrm{mg}$. Nei pazienti anziani, a causa di una ridotta velocità di metabolizzazione, sono state riscontrate un'emivita più lunga e una clearance diminuita. come altri farmaci psicotropi, citalopram si distribuisce nell'organismo; le concentrazion più elevate di farmaco e di metaboliti demetilati si trovano nei polmoni, nel fegato, nei reni, concentrazioni inferiori nella milza, nel cuore e nel cervello. Il farmaco e i suoi metaboliti passano la barriera placentare e si distribuiscono nel feto in modo simile a quanto visto nella madre. Una piccolissima quantità di citalopram e dei suoi metaboliti sono secreti nel latte materno. Citalopram è metabolizzato a demetilcitalopram, didemetilcitalopram, citalopram $\mathrm{N}$-ossido e, per deaminazione, a un derivato deaminato dell' acido propionico. Mentre il derivato dell'acido propionico è inattivo, demetilcitalo pram, didemetilcitalopram e citalopram $\mathrm{N}$-ossido, sono pure inibitori selettivi dell'uptake della serotonina, sebbene più deboli del composto di origine. Nei pazienti, il citalopram non metabolizzato è il composto predominante nel plasma. II rapporto di concentrazione citalopram/dimetilcitalopram nel plasma, allo stato stazionario, è mediamente di 3,4 dopo 15 ore e di 2 dopo 24 ore dalla somministrazione. I livelli plasmatici di didemetilcitalopram e citalopram $\mathrm{N}$-ossido sono generalmente molto bassi. Non è stata effettuata una valutazione tra concentrazione plasmatica ed effetto, nemmeno gli effetti collaterali sembrano correlati alle concentrazioni plasmatiche del farmaco. La clearance plasmatica sistemica è pari a circa $0,4 \mathrm{l} / \mathrm{min}$ 'escrezione awiene con le urine e con le feci. II fattore di conversione da nM a ng/ml (riferito alla base) è 0,32 per il citalopram e 0,31 per il demetilcitalopram.
5. INFORMAZIONI CLINICHE.

L'indicazione per citalopram è la sfera del disordini depressivi endogeni. Citalopram non interferisce sul sistema di conduzione cardiaca o sulla pressione arteriosa. Questo è particolarmente importante per i pazienti anziani. Inoltre citalopram non ha effetti sui sistemi ematico, epatico o renale. La frequenza degli effetti collaterali è molto bassa e la maggior parte dei sintomi è di lieve entità e di tipo transitorio. Gli effetti collaterali osservati con maggior frequenza sono nausea e aumentata sudorazione. Dopo poche settimane di trattamento la maggior parte dei pazienti non ha manifestato alcun effetto collaterale. La bassa frequenza di effetti collaterali e le minime proprietà sedative di citalopram ne suggeriscono l'impiego nei trattamenti a lungo termine. inoltre citalopram non causa aumento del peso corporeo né potenzia l'effetto dell'alcool. 5.1. Indicazioni terapeutiche. Sindrom depressive endogene. 5.2. Controindicazioni. Ipersensibilità verso i componenti del prodotto. Età inferiore ai 14 anni. Citalopram non deve essere somministrato a pazienti in trattamento con MAO-inibitori e comunque non prima di 14 giorni dopo la loro sospensione (vedere Awertenze). 5.3. Effetti indesiderati. Citalopram è generaimente ben tollerato; le reazioni secondarie osservate sono in generale poche, di lieve entità e di tipo transitorio. Le reazioni che compaiono con maggiore frequenza sono nausea aumentata sudorazione, secchezza delle fauci, cefalea e ridotta durata de sonno. Esse si manifestano soprattutto nella prima o seconda settimana di terapia per poi sparire con il miglioramento dello stato depressivo $\mathbf{5 . 4}$. Speciall precauzioni per l'uso. Citalopram non deve essere somministrato a pazienti in trattamento con MAO-inibitori e comunque non prima di almeno 14 giorni dopo la loro sospensione. Nei pazienti con insufficienza epatica è consigliabile una dose giornaliera non superiore a 20-30 mg. Nei pazienti con funzionalità renale fortemente ridotta è consigliabile atteners al dosaggio minimo consigliato. 5.5. Uso in caso di gravidanza e allattamento. L'innocuità di citalopram in gravidanza non è stata stabilita. Sebbene gli studi effettuati sugli animali da esperimento non abbiano evidenziato segni di potenziale teratogenicità, né effetti sulla riproduzione o sulle condizioni perinatali, poiché il citalopram con i suoi metaboliti passa la barriera placentare e poiché una piccolissima quantità viene riscontrata nel latte materno, se ne sconsiglia l'uso durante la gravidanza e l'allattamento. 5.6. Interazioni medicamentose e altre. La somministrazione contemporanea di MAO-inibitori può causare crisi ipertensive (vedere Awertenze) Non sono state riportate interazioni legate alla assunzione contemporanea dell'alcool. Livelli plasmatici di citalopram leggermente elevati sono stati osservati durante il trattamento contemporaneo con alcune fenotiazine (levomepromazina e alimemazina) ma il dato non ha rilevanza clinica Benché non esistano dati relativi aila possibile inibizione del citalopram da parte della cimetidina i pazienti in trattamento con questo farmaco devono ricevere dosaggio dimezzato ed essere attentamente monitorati. 5.7 Posologia e modalità di somministrazione. Adulti: Seropram compres se viene somministrato in un'unica dose giornaliera. La dose iniziale è di 20 $\mathrm{mg}$ al giorno, la sera. Questa può essere aumentata a $40 \mathrm{mg}$ al giorno, se necessario. Anziani: Ai pazienti sopra i 65 anni di età deve essere somministrata metà della dose raccomandata a causa di un rallentato metabolismo. Bambini: Non esistono esperienze cliniche circa l'impiego pediatrico di citalopram; pertanto se ne sconsiglia la somministrazione a pazienti con età inferiore a 14 anni. L'effetto antidepressivo si manifesta in genere entro 2-4 settimane dall'inizio della terapia' è opportuno che il paziente venga seguito dal medico fino a remissione dello stato depressivo. Poiché il trattamento con antidepressivo è sintomatico, deve essere continuato per un appropriato periodo di tempo, in genere 4-6 mesi nelle malattie maniaco-depressive In caso di insonnia o di forte irrequietezza si raccomanda un trattamento addizionale con sedativi in fase acuta. 5.8. Sovradosaggio. I sintomi possibili con una dose fino a $600 \mathrm{mg}$ sono: stanchezza, debolezza, sedazione vertigini, tremori delle mani, nausea. I sintomi possibili con dosi superior a $600 \mathrm{mg}$ sono: torpore è disturbi respiratori ma non segni di cardiotossicità. La dose più alta ingerita è stata di $2000 \mathrm{mg}$ : il paziente è stato ricoverato in condizioni stuporose e con difficoltà respiratorie, ma senza evidenza di cardiotossicità. II paziente si è comunque ripreso rapidamente. ॥ trattamento del sovradosaggio è sintomatico e di supporto in quanto non esiste un antidoto specifico' una lavanda gastrica dovrebbe essere effettuata non appena possibile dopo l'ingestione orale e la pervietà delle vie aeree deve essere mantenuta, se necessario con intubazione Somministrare ossigeno in caso di ipossia e diazepam in caso di convulsioni. Ė consigliabile la sorveglianza medica per circa 24 ore. Non esiste uno specifico antidoto ma il paziente può trarre beneficio dalla somministrazione di antagonisti della serotonina (es. metisergide). 5.9. Avvertenze. Non somministrare a pazienti di età inferiore a 14 anni. Qualora il paziente entrasse in una fase maniacale, il trattamento deve essere sospeso e si deve istituire un trattamento appropriato con neurolettici Il rischio di suicidio nei pazienti depressi persiste fino a quando non si ottiene una significativa remissione, poiché il blocco inibitorio può venire meno prima che si stabilisca una efficace azione antidepressiva. 5.10. Effetti sulla capacità di guidare e sull'uso di macchine. Citalopram ha scarsi effetti sulla performance psicomotoria.

\section{INFORMAZIONI FARMACEUTICHE.}

6.1. Incompatibilità. Nessuna 6.2 Durata di stabilità a confezionamento integro. 5 anni. 6.3. Speciali precauzioni per la conservazione. Nessuna. 6.4. Contenitore, confezione e relativi prezzi. Le compresse vengono confezionate in blister di PVC e alluminio. 28 compresse $20 \mathrm{mg}$ Lit. 67.000. 6.5. Sede sociale del titolare dell'autorizzazione all'immissione sul mercato. Lundbeck Italia S.p.A. - via Fara, 35 20124 Milano. Officina di produzione: H. Lundbeck A/S Copenhagen valby (Danimarca) 6.6. Numero di codice e data di prima commercializzazione. 28 compresse rivestite $20 \mathrm{mg} n$. di codice: 028759013 . Prima commercializzazione: Marzo 1995. 6.7. Eventuale tabella di appartenenza. Nessuna. 6.8. Regime di dispensazione al pubblico. Su presentazione di ricetta medica. 
I I trattamento dell'ansia e della depressione. Guida all'uso razionale dei farmaci nella medicina generale. Un libro di Cesario Bellantuono, dell'Istituto di Psichiatria dell'Università di Verona, membro dello European

College of

Psychopharmacology.

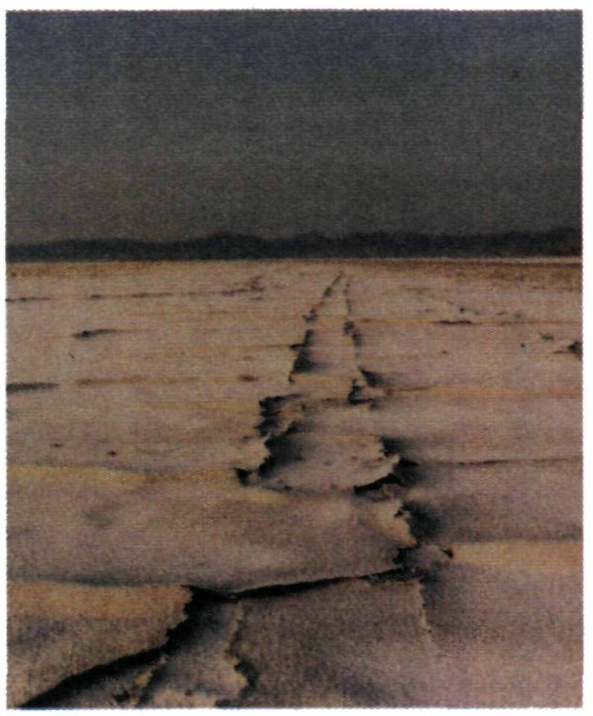

Nutrizione e

tumori. Un libro di

Carlo La Vecchia, capo del Laboratorio di Epidemiologia dell'Istituto Mario Negri, docente di Epidemiologia presso l'Università di Milano e la Harvard School of Public Health. Editor dello European Journal of Public Health e del Journal of Epidemiology and Biostatistics.

\section{una nuova collana di libri tascabili del Pensiero Scientifico Editore. Per non sostare e lasciare il segno.}
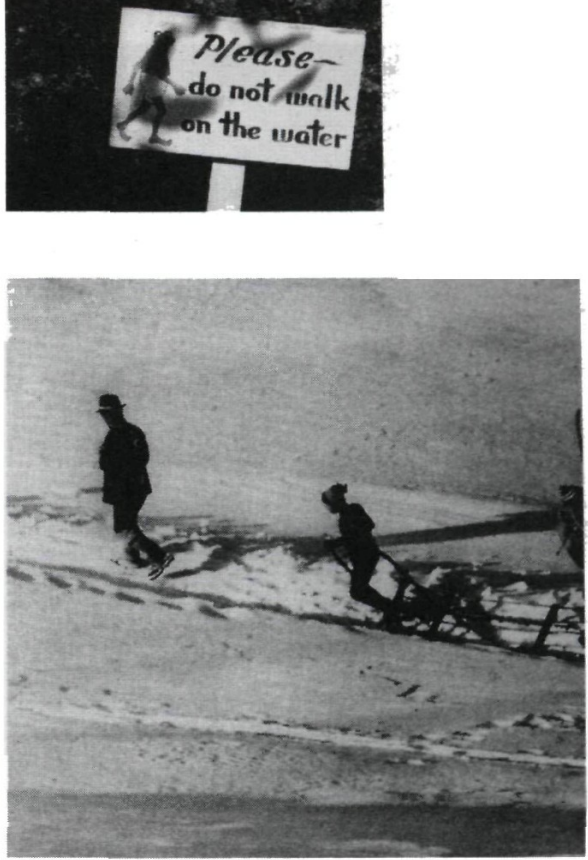

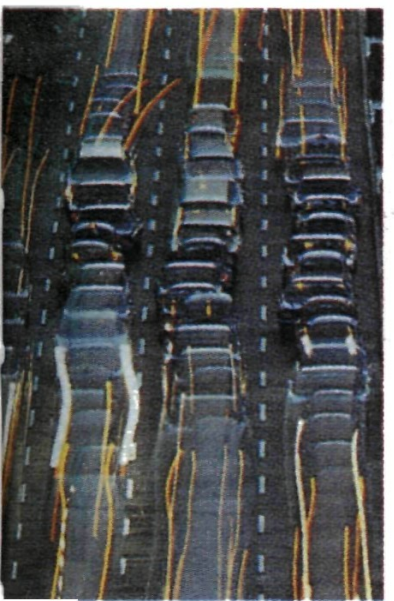

T umori e qualità di vita. Un libro di Giovanni Apolone e Paola Mosconi, della Unità di Valutazione degli interventi sanitari dell'Istituto Mario Negri, di Fausto Roila, della Divisione di

Oncologia medica dell'Università di Perugia e di Enzo Ballatori del Dipartimento di Medicina Interna e Salute pubblica dell'Università di L'Aquila.

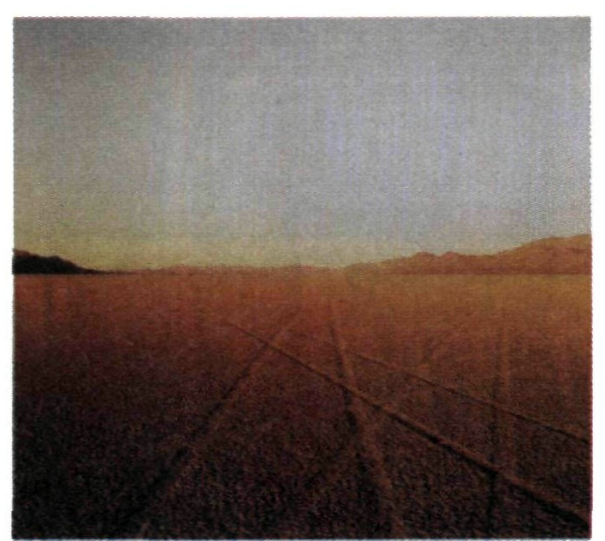

\section{Morbo di}

Parkinson: la qualità di vita del malato in relazione alle strategie terapeutiche. Un libro di Fabrizio Stocchi, della Clinica Neurologica dell'Università di Roma, membro della Movement Disorders Society, della European Neurological Society e della American Academy of Neurology.
I costi dell'assistenza psichiatrica.

Un libro di Francesco Amaddeo, Paola Bonizzato e Michele Tansella, della Unità di Valutazione dei costi in Psichiatria (Università di Verona), che svolge ricerche in collaborazione con il centro di Health Economics dell'Istituto di Psichiatria della Università di Londra.

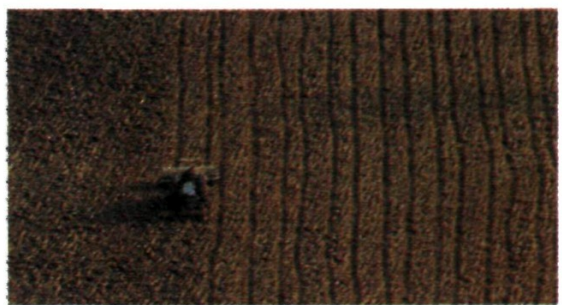




Recupero a tuttigli effetti.

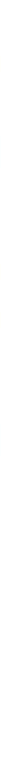

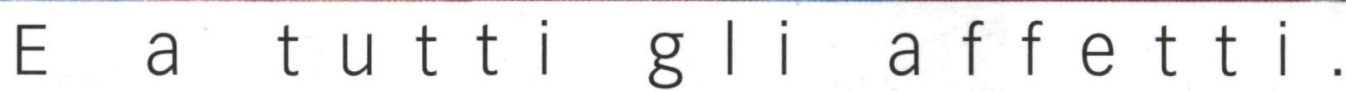

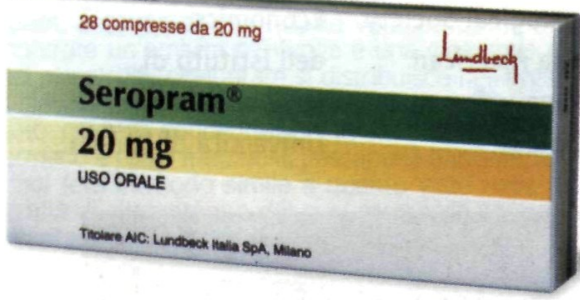

"L'evidenza di efficacia di citalopram, nella prevenzione delle ricadute, risulta saldamente documentata attraverso i risultati positivi di due studi prospettici" ${ }^{\prime(1)}$

(1) Robert Ph, Montgomery SA, Internat Clin Psychopharmacol, 10 (Suppl 1): 29-35; 1995.

(2) Montgomery SA et al. Internat Clin

Psychopharmacol, 8: 181-188; 1993.
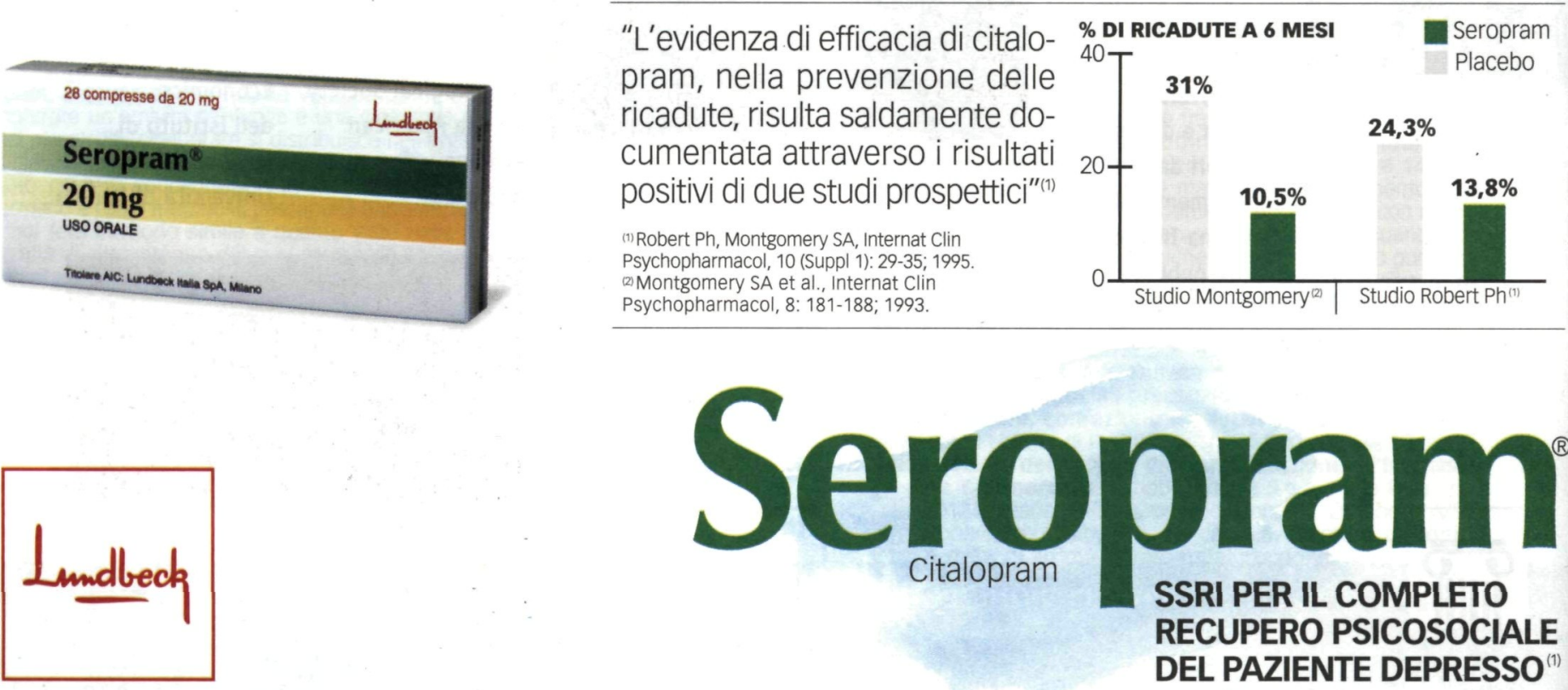